\title{
Chlorotoxin Redirects Chimeric Antigen Receptor T Cells for Specific and Effective Targeting of
}

\section{Glioblastoma}

Authors: Dongrui Wang ${ }^{1,4}$, Renate Starr ${ }^{1}$, Wen-Chung Chang ${ }^{1}$, Brenda Aguilar ${ }^{1}$, Darya Alizadeh ${ }^{1}$, Sarah L. Wright ${ }^{1}$, Xin Yang ${ }^{1}$, Alfonso Brito ${ }^{1}$, Aniee Sarkissian ${ }^{1}$, Julie R. Ostberg ${ }^{1}$, Yanhong Shi $^{3}$, Margarita Gutova $^{3}$, Karen Aboody ${ }^{3}$, Behnam Badie ${ }^{2}$, Stephen J. Forman ${ }^{1, \#}$, Michael E. Barish ${ }^{3, \#}$, Christine E. Brown $^{1, \#, *}$

\section{Affiliations:}

${ }^{1}$ Department of Hematology \& Hematopoietic Cell Transplantation, T Cell Therapeutics Research Laboratory; ${ }^{2}$ Division of Neurosurgery, Department of Surgery; ${ }^{3}$ Department of Developmental and Stem Cell Biology; ${ }^{4}$ Irell and Manella Graduate School of Biological Sciences; City of Hope Beckman Research Institute and Medical Center, Duarte, CA 91010.

${ }^{\#}$ Co-senior authors

*Correspondence should be addressed to C.E.B. (cbrown@coh.org)

One Sentence Summary: Chimeric antigen receptors incorporating chlorotoxin as the tumor targeting domain recognize and kill glioblastoma with high specificity and potency.

\begin{abstract}
While chimeric antigen receptor (CAR) T cells have demonstrated antitumor activity against glioblastoma (GBM), tumor heterogeneity remains a critical challenge. To more effectively target heterogeneous GBMs, we report the development of a novel peptide-based CAR exploiting the GBM-binding potential of chlorotoxin (CLTX). CLTX bound a greater proportion of tumor cells than GBM-associated antigens EGFR, HER2 and IL13R $\alpha 2$. CAR T cells bearing CLTX as the targeting domain (CLTX-CAR), mediated potent in vitro and in vivo anti-GBM activity, and efficiently targeted tumors lacking expression of other
\end{abstract}


GBM-associated antigens. Importantly, CLTX-CAR T cells exhibited no observable off-target effector activity against normal cells, or when adoptively transferred into mice. Effective targeting by CLTX-CAR T cells required cell surface expression of matrix metalloproteinase-2 (MMP-2). Our results are the first demonstration of a peptide toxin utilized as a CAR targeting domain, expanding the repertoire of tumorselective CAR T cells with the potential to reduce antigen escape.

\section{Introduction}

Glioblastoma (GBM) is the most common type of primary brain tumor. Despite the increasingly aggressive treatments incorporating surgery, chemotherapy and radiotherapy, the survival of GBM patients has been only modestly improved over the last several decades $(1,2)$. The poor prognosis for patients with GBMs has prompted the development of advanced therapies, among which is immunotherapy using $\mathrm{T}$ cells engineered with chimeric antigen receptors (CARs) $(3,4)$. CAR T cell therapy redirects the cytotoxic activity of T lymphocytes independent of MHC restriction and without need for antigen priming. This cellular therapy, therefore, provides a strategy to generate de novo antitumor immunity, which may overcome the challenges of low mutational burden and lack of immunogenicity in tumors such as GBMs with low mutational burdens (5, 6). We and others have demonstrated that CAR T cell therapy can be successfully translated for the treatment of GBM (7-10), demonstrating safety, evidence for antitumor activity and in one case the potential for mediating complete tumor remission (8).

Despite encouraging evidence of clinical safety and bioactivity for GBM-targeted CAR T cells, the overall response rates have been unsatisfyingly low, especially as compared to the remarkable clinical responses observed against $\mathrm{B}$ cell malignancies $(11,12)$. One of the major obstacles complicating CAR T cell therapeutic efficacy is tumor heterogeneity, which is particularly significant in GBMs. The classification of GBM subtypes has illustrated the heterogeneity across patients, and more recent studies using single cell sequencing also revealed considerable genetic variations among intratumoral 
subpopulations $(13,14)$. Efforts to develop CAR T cells for GBMs, therefore, must consider this high degree of heterogeneity. We have demonstrated that IL13 receptor $\alpha 2$ (IL13R $\alpha 2$ ) expression is associated with a mesenchymal phenotype and poorer prognosis of GBM patients (15). For IL13Ra2-positive tumors, its expression has also been verified on tumor-initiating glioma stem-like cells, and expression is associated with tumor invasiveness (16). However, after treating with IL13R $\alpha 2$-targeted CAR T cells, instances of tumor recurrence with loss and/or reduced expression of IL13R $\alpha 2$ has been observed $(8,17)$. Similar results were also reported following EGFR variant III (EGFRvIII)-targeted immunotherapies, with the down-regulation of EGFRvIII expression in the tumors harvested post-therapy $(10,18)$. Indeed, GBMs are able to rapidly adapt to therapies, resulting in relapse with distinct intratumoral cellular profiles (19). Given the promising clinical results of CAR therapy against B cell malignancies by targeting CD19 which is broadly expressed by all B cell lineages (20-22), the development of more effective GBMtargeting CAR designs is expected to benefit from improving broad tumor recognition. To date, this has remained elusive due to the scarcity of antigen candidates that are both widely expressed and highly tumor-specific, especially given the extreme risk of off-tumor toxicities due to the critical location of these tumors within the brain.

An opportunity to extend the repertoire of target antigens amenable to CAR T cell therapy is presented by the tumor-binding potential of some naturally-derived molecules (23). One example is chlorotoxin (CLTX), a 36-amino acid peptide isolated from the venom of the death stalker scorpion (24). The GBMbinding potential of CLTX was first identified through conjugation with the radioisotope ${ }^{131} \mathrm{I}(25)$. While the precise cell surface receptor for CLTX on GBM cells remains unclear, CLTX binding impairs GBM cell migration and invasiveness $(26,27)$. Importantly, despite the tumor-binding potential, CLTX itself elicits minor toxic reactions against both tumor and normal tissues $(28,29)$. Therefore, one line of research has been focused on incorporating other cytotoxic agents with CLTX, aiming for tumor-specific delivery. An early clinical study using ${ }^{131}$ I-labeled CLTX successfully mediated radiotherapy against post-resection residual tumor without major neurotoxicity (28). CLTX has also been used to coat a variety 
of delivery vehicles to administer chemotherapy drugs as well as small interfering RNAs (siRNAs) (30, 31). More recently, the potential of CLTX to deliver fluorescent signal specifically to tumors (named “Tumor Paint”) has been demonstrated in preclinical models and is currently under clinical investigation (29, 32, 33). These studies have indicated that CLTX can be manipulated and harnessed for specific tumor targeting.

Here we report the preclinical development of a novel peptide-based CAR using CLTX as the tumortargeting domain. CLTX specifically bound a panel of primary GBM cells, with a more inclusive pattern than expression of other GBM-associated antigens. We show that CLTX-CAR T cells incorporating an IgG4(EQ) spacer and a CD28 co-stimulatory signal, were active against GBMs with distinct phenotypes, and elicited antitumor immune responses without major off-tumor toxicities in mouse models with human GBM xenografts. Therefore, our results suggest that CLTX-CAR T cells have the potential to effectively mediate GBM eradication, and represent an advanced approach to CAR design for broad and specific tumor targeting.

\section{Results}

\section{CLTX binds to a broad variety of GBM cells.}

Previous studies have documented the GBM-selective binding properties of CLTX (25, 34). However, CTLX binding, in relation to either other tumor associated antigens or to GBM subpopulations, has not been examined. We first evaluated CLTX binding to freshly-dissociated tumor cells from surgical resection specimens. These primary brain tumor (PBT) cells were examined by flow cytometry for binding of Cy5.5-conjugated CLTX peptide (CLTX-Cy5.5) and compared with expression of IL13R $\alpha 2$, HER2 and EGFR, three targets being clinically evaluated in CAR T cell therapies for GBMs $(10,17,35)$. Strong CLTX-Cy5.5 fluorescence was observed for almost all patient tumors, with greater than $80 \%$ of cells binding CLTX (Fig. 1A and 1B, left panel). Across 22 tumor samples from 15 different patients, only two (PBT114 and PBT131) showed CLTX-Cy5.5 binding in less than 30\% of total cells. At the 
same time, expression of immunotherapy targets IL13R $\alpha 2$, HER2 and EGFR varied widely between patient tumors. CLTX-Cy5.5 binding appeared independent of other antigens, and was observed on tumors with both high and low expression of IL13R $\alpha 2$, HER2 and EGFR (See representative flow cytometry plots in Fig. 1A). We also examined CLTX-Cy5.5 binding to low-passage patient-derived GBM tumor sphere (PBT-TS) lines expanded under conditions favoring a cancer stem cell-like phenotype (36-38). Similar to dissociated primary GBM cells, 18 out of 19 PBT-TS lines showed greater than 80\% CLTX-Cy5.5 binding (Fig. 1B, right panel and Fig. S1A), including the TS lines which displayed negligible expression of IL13R 2 2, HER2 and EGFR (PBT003-4-TS, PBT009-TS). To evaluate CLTX binding in engrafted tumors, GBM orthotopic xenografts were tested by fluorescent microscopy using a biotin-conjugated CLTX peptide. Consistent with the analyses of freshly resected patient tumors, CLTX displayed consistent binding to five of five engrafted PBT-TS GBM tumors, and marked a greater proportion of tumor cells as compared to the expression of IL13R 22 and EGFR (Fig. 1C and Fig. S1B). Taken together, these studies confirmed the capacity of CLTX to bind to a high percentage of patient GBM tumors (20 of 22 freshly-dissociated GBM samples), as well as to the majority of GBM cells within each tumor.

Cells within GBM tumors are highly heterogeneous, composed of phenotypically and functionally distinct subpopulations. Within GBM tumors, stem-like cells (GSCs) display self-renewal and tumor-initiation capacity (39), identifying this population as a therapeutically important component of a GBM-targeted CAR T cell therapy $(16,35,40)$. Hence, we examined CLTX binding with respect to this stem cell-like population. First in freshly-dissociated primary PBTs, we distinguished GSCs from other GBM cells by expression of surface markers CD133 or CD44 $(41,42)$. In the aggregate, we observed that CLTX-Cy5.5 binding was somewhat higher in CD133+ GSCs, but also evident in more differentiated CD133- cells, whereas no significant difference was observed between CD44+ and CD44- GBM cells (Fig. 1D). In another approach, we used PBT-TS lines and varied culture conditions to favor GSCs or alternatively to promote differentiation (16). Differentiation led to reduced expression of GSC-marker CD133; however, 
CLTX-Cy5.5 binding was not affected in comparison to GSCs (Fig. 1E). These studies demonstrated that CLTX binding, while showing some preference for CD133+ GSCs in freshly dispersed tumor samples, remains robust on both stem-like and more differentiated GBM cells. Together, these studies demonstrated the broad GBM binding potential of CLTX, providing rationales for investigating its use to redirect CAR T cell immunotherapy.

CLTX-CAR T cell potency can be optimized through modifying non-targeting domains

We next sought to design a CAR incorporating the CLTX peptide as the tumor targeting domain. The initial CLTX-CAR was generated using the backbone of our CD19-targeted CAR that has shown safety and clinical activity against B-cell malignancies (43). This CLTX-CAR construct is comprised of the CLTX peptide, an IgG4Fc(EQ) spacer, and a CD28 costimulatory domain (Fig. 2A), and is referred to as

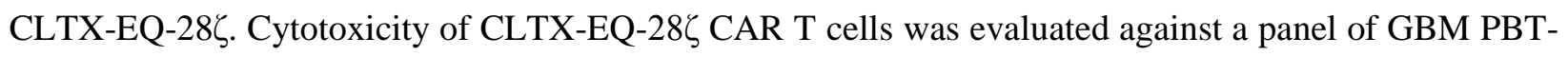
TS lines. CLTX-EQ-28ל CAR T cells conjugated with tumor cells within 2 hours and formed immunological synapse-like structures (Fig. 2B). Co-culture with GBM cells stimulated CLTX-EQ-28ל CAR T cells to up-regulate T cell activation markers CD69 and 4-1BB (CD137), and to degranulate as measured by cell-surface CD107a expression (Fig. 2C and Fig. S2A). Further, CLTX-EQ-28ל CAR T cells efficiently killed GBM cells in 48-hour co-culture assays at an effector to target (E:T) ratio of 1:4

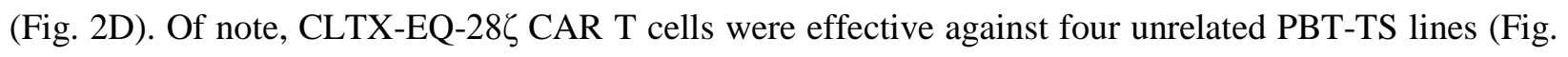
2D) that exhibited distinct IL13Ra2, HER2 and EGFR expression profiles (Fig. 1B and Fig. S1A). In particular, while IL13R $\alpha 2$-targeted CAR T cells failed to respond to the PBT-TS lines with low/negative

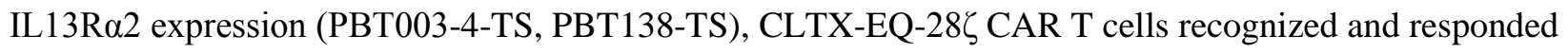
to all four TS lines (Fig. 2, B-D). Further, focusing on PBT003-4-TS, which showed negligible expression of IL13R $\alpha 2$, HER2 or EGFR (Fig. S1A), we demonstrated that co-culture with CLTX-EQ-28ל, but not the combination of CAR T cells targeting all three other antigens, mediated tumor cell elimination (Video.S1 and S2). Lentiviral transduction of PBT003-4-TS to over-express IL13R $\alpha 2$, HER2 or EGFR did not reduce CLTX-EQ-28ร cytotoxic activity (Fig. S2, B and C). We thus concluded that CLTX-EQ- 
$28 \zeta$ was able to recognize and target GBM cells, and its cytotoxicity is consistent with the broad GBMbinding potential of the CLTX peptide while independent of other GBM-associated antigens.

The cytolytic activity of CAR T cells is greatly influenced by the design of regions outside of the antigentargeting domain, including the spacer (44) and the co-stimulatory domains (45-47). Using the CLTXEQ-28 CAR as a reference, we first addressed the impact of spacer length by generating CAR constructs to compare IgG4Fc(EQ) (239 amino acids) with three shorter spacers: IgG4-Fc with the CH2-domain deleted ( $\triangle \mathrm{CH} 2)$ (129 amino acids), CD8 hinge (CD8h) (44 amino acids), and a short synthetic linker (L) (10 amino acids) (Fig. 3A). We observed that CAR T cell-mediated tumor killing was greatly reduced with $\triangle \mathrm{CH} 2$ or L spacers, while the CD8h spacer retained CAR function similar to that of IgG4Fc(EQ) (Fig. 3B). We next evaluated the contributions of co-stimulatory signals by generating CARs bearing CD28 or 4-1BB co-stimulatory domains in the contexts of spacers IgG4Fc(EQ) and CD8h (Fig. 3A). Intriguingly, CAR T cells incorporating a CD28 co-stimulation domain consistently showed higher T cell effector function as compared with CARs incorporating a 4-1BB co-stimulation domain (Fig. 3C). We then evaluated the mechanism underlying the functional differences across CLTX-CAR constructs. Notably, for the six CLTX-CAR constructs evaluated, we observed consistent tumor-dependent activation across multiple assays including killing potency (\% killing), extent of degranulation (\% CD107a+), and secretion of cytokine IFN $\gamma$ (Fig.3, B and C, and Fig. S3A). Moreover, consistent with the potent cytotoxic

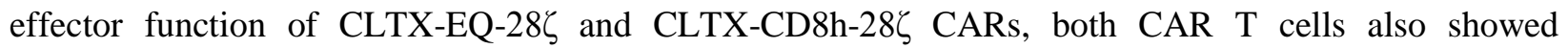
expression of T cell activation markers 4-1BB and CD69 (Fig. S3B), and inhibitory molecule PD-1 which is also linked to T cell activation (Fig. S3C). These results therefore suggested that differences in initial activation were the major contributor to variations in effector function across various CLTX-CAR designs.

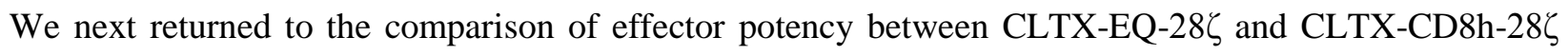
CAR T cells. First, screening degranulation and cytokine-production against a panel of 10 PBT-TS lines,

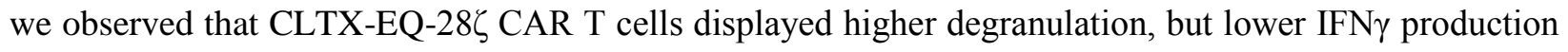

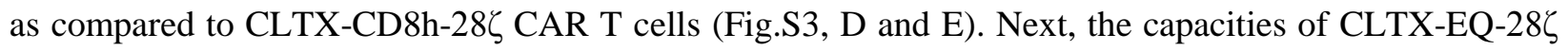


and CLTX-CD8h28 CAR T cells to maintain long-term antitumor activity were evaluated in a repetitive

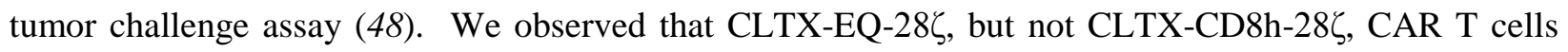
retained activity through multiple rounds of tumor challenge (Fig. 3D). Since both CLTX-EQ-28ร and

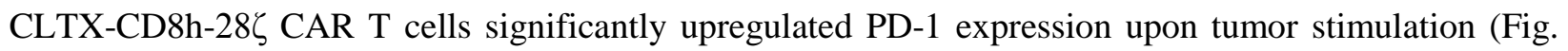
S3C), we then assessed co-expression of three $\mathrm{T}$ cell inhibitory receptors (PD-1, LAG-3 and TIM-3) which together marks exhausted $\mathrm{T}$ cells $(48,49)$, and observed that the absence of durable effector function in CLTX-CD8h-28ל CAR T cells was associated with an exhausted phenotype featuring coexpression of multiple inhibitory receptors (Fig. 3E). Considering all of these observations about effector potency, we adopted the CLTX-EQ-28ל CAR as the optimal design for subsequent evaluation.

\section{CLTX-CAR T cells mediate antitumor activity against established GBM xenografts}

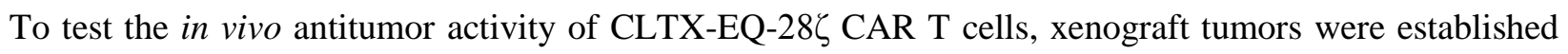
using two patient-derived PBT-TS lines with distinct antigen expression patterns: PBT003-4-TS and PBT106-TS (Fig. S1A). Against subcutaneously engrafted GBMs, intratumoral administration of CLTXEQ-28 CAR T cells resulted in tumor regression, while tumors injected with mock-transduced T cells displayed growth kinetics similar to tumor-only controls (Fig. S4A). Next, the antitumor function of CLTX-EQ-28ל CAR T cells was evaluated in orthotopic GBM models in which PBT-TS lines were stereotactically implanted into the brains of immunodeficient mice. After engraftment, the brains were subjected to intracranial injection of CAR T cells (50). Consistently with the subcutaneous tumor model,

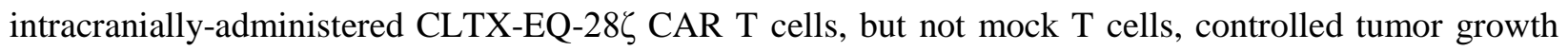
and prolonged the survival of mice bearing PBT003-4-TS or PBT106-TS tumors (Fig. 4, A-D).

Of note, the responses of engrafted PBT003-4-TS and PBT106-TS tumors to CLTX-CAR T cell therapy were not equivalent. After CAR T cell administration, all mice bearing PBT106-TS tumors remained tumor-free for over 170 days, while only a subset of PBT003-4-TS tumor-bearing mice achieved similar long-term tumor eradication (Fig. 4A-D and Fig. S4B). To investigate the etiology of this variable tumor 
response against different GBM models, we examined recurrent PBT003-4-TS tumors post CLTX-EQ$28 \zeta$ therapy. We first observed that CLTX-Cy5.5 binding on these recurrent tumors was similar to untreated tumors (Fig. 4E), suggesting that antigen escape did not account for tumor relapse. Investigating the behavior of antitumor $\mathrm{T}$ cells within these orthotopic tumors, we found that during primary antitumor response, granzyme B-expressing $\mathrm{T}$ cells were detected 14 days following adoptive transfer (Fig. 4F top

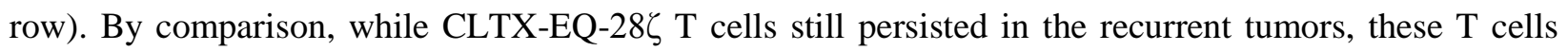
expressed only low levels of granzyme B (Fig. 4F bottom row). At the same time, these relapsed PBT003-4-TS tumors displayed increased expression of PD-L1 as compared to untreated tumors (Fig. 4G). PD-L1 induction on tumors post CAR treatment was consistent with a high IFN- $\gamma$ receptor A (IFN $\gamma$ RA) expression in GBM cells from PBT003-4-TS before implantation (Fig. 4H). IFN- $\gamma$ had been shown to promote PD-L1 transcription through its receptors in other tumors (51), and IFN- $\gamma$ treatment rapidly induced PD-L1 expression by PBT003-4-TS cells (Fig. 4I). Notably, cells from PBT106-TS exhibited lower levels of IFN $\gamma$ RA expression and less robust PD-L1 induction by IFN- $\gamma$ treatment, as compared to PBT003-4-TS (Fig. 4, H and I). These observations suggested that for some GBMs, adaptive response to immunostimulatory cytokines in tumors and subsequent induction of inhibitory checkpoint receptors, rather than antigen escape, was the main reason for insufficient tumor eradication by CLTXCAR T cells.

\section{CLTX-CAR T cells have minimal off-target effects}

Prior clinical studies using CLTX to deliver radiation and imaging reagent to tumor sites (NCT00040573, NCT02234297) have not reported significant adverse events, and multiple preclinical studies have shown no off-target toxicity $(29,32)$. Consistent with these reports, we observed limited to undetectable CLTXCy5.5 binding to a panel of non-tumor cells, including periphery blood mononuclear cells (PBMCs), human embryonic kidney 293T cells, as well as induced pluripotent stem cell-differentiated astrocytes (iPSC-Ast), neural progenitor cells (iPSC-NPCs) and immortalized fetal brain derived neural stem cell (FB-NSC) line LM-NSC008 (52-54) (Fig. 5A). We next concerned that even a low-level of binding could 


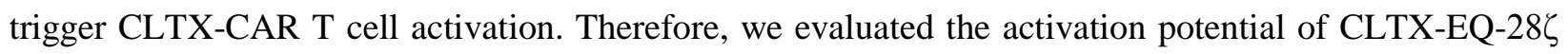
CAR $\mathrm{T}$ cells during co-culture with these normal cells. As indicated by degranulation and in vitro

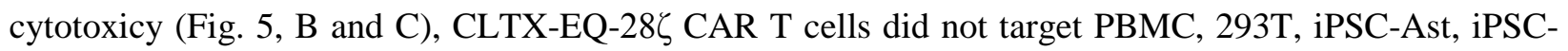
NPC and FB-NSC, exhibiting only background levels of effector activity equivalent to mock transduced T cells. Further, the observation that CLTX-Cy5.5 binding is conserved between mouse and human GBM cells (see Fig. 5D for examples of CLTX-Cy5.5 binding to mouse KR158 and GL261 GBM cells) supports the relevance of murine models for assessing CLTX-CAR T cell off-tumor toxicities. In mouse brains bearing GBM xenograft tumors, CLTX-Cy5.5 bound to tumor cells, but not normal brain tissue, delineating the xenograft-tumor border (Fig. 5E). In addition, intratumoral administration of CLTX-EQ-

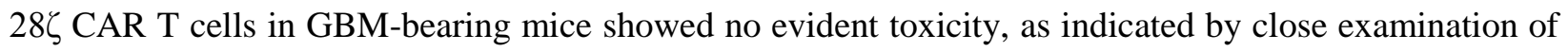
multiple organs including brain, kidney, liver, spleen, intestine and colon (Fig. S5A). The potential for

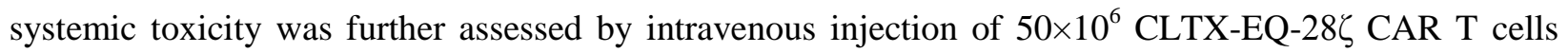
into normal NSG mice. At this high dose, CLTX-CAR T cells were well-tolerated; all animals remained alert and active, and showed no changes in body weight (Fig. 5F). Taken together, these observations

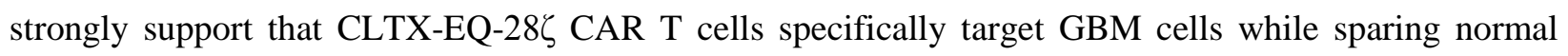
tissues.

MMP2-expression is required for CLTX-CAR targeting

CLTX binding has been reported to associate with multiple membrane proteins, including membrane bound matrix metalloproteinase 2 (MMP2), chloride channel CLCN3, and phospholipid protein Annexin A2 (AXNA2) $(26,27,55)$. With the composition of the CLTX receptor poorly identified, we took advantage of CLTX-CAR T cells to investigate the correlation between the expression of these surface proteins on GBM cells and CLTX-CAR effector activity. Using 15 different PBT-TS lines and iPSC-

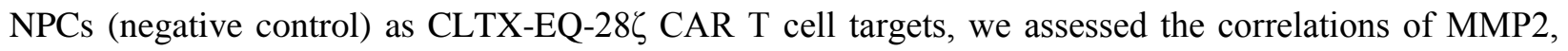
CCLN3, and AXNA2 expression with CLTX-CAR T cell activity (degranulation potential). We found that CLTX-EQ-28ל CAR T cell activity varied between these different GBM target cells (Fig. 6A), and 
observed a strong correlation between CLTX-CAR T cell degranulation and target cell MMP2 expression (Fig. 6B), but not with expression of CLCN3 or ANXA2 (Fig. S6A). Consistent with this pattern, CLTXCy5.5 binding correlated with MMP2 expression on target cells (Fig. 6C), but not with CLCN3 or AXNA2 expression (data not shown). Differences in CLTX-CAR cytotoxic potential against different GBM cells were most evident at an E:T ratio of 1:8 (Fig. S6B), and correlated well with degranulation (Fig. S6C). Importantly, the extent of degranulation did not correlate with IFN $\gamma R A$ (CD119) expression on PBT-TS lines (Fig. S6D), which was previously related to low in vivo CLTX-CAR T cell function through induction of PD-L1. These results suggest that for GBM cells, MMP2 is the primary mediator of CLTX-Cy5.5 binding and CLTX-CAR T cell cytotoxicity. To further verify the necessity of MMP2 for CLTX-CAR activation, we knocked down MMP2 in GBM cells using lentiviral shRNA. The knockdown dramatically reduced MMP2 transcript levels and MMP2 secretion, and also resulted in modest decreases in CLCN3 expression and minimal changes in ANXA2 expression (Fig. S6E). When subjected to in vitro co-culture, we found that CLTX-EQ-28ל CAR T cell activation and cytotoxicity were dramatically reduced by shMMP2 transduction (Fig.6, D and E, Fig. S6F). Further, MMP2 knockdown significantly

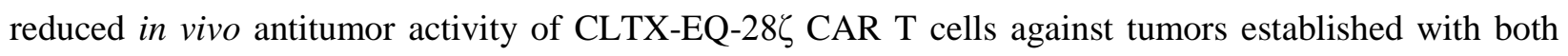
PBT003-4-TS and PBT106-TS (Fig. 6F). Together, these results demonstrate that MMP2 is necessary for CLTX-CAR recognition and activation against tumor targets.

MMP2 is a secreted matrix metalloproteinase that can either associate with the membrane through interaction with $\alpha_{v} \beta_{3}$ integrin and MMP14 [also known as membrane-type 1 (MT1)-MMP], or exist as a soluble enzyme $(26,56)$. Although our data and others indicate that CLTX binds to the membrane-bound form of MMP2 (26), we further investigated whether CLTX-CAR T cells may also respond to soluble MMP2. In the presence of soluble MMP2, we observed no activation of CLTX-CAR T cells (Fig. 6G), and the cytotoxicity of CLTX-CAR T cells against GBM cells was also maintained (Fig. 6, G and H). In addition, we visualized the recruitment of MMP2 to the CLTX CAR T cell-GBM cell immunological synapse (Fig. 6I) but not at the synapse formed by other CAR T cells (Fig. S6G). Meanwhile, we were 
intrigued to find that CLCN3, although not correlated with CLTX-CAR T cell activation, was also recruited to the immunological synapse (Fig. 6I). Similarly, we observed co-localization of MMP2 and CLCN3 when CLTX peptides were applied to GBM cells (Fig. S6H). These results suggest that binding of CLTX-CAR T cells to a GBM cells depends on expression of MMP2 and involves CLCN3. Recruitment of CLCN3 following MMP2 interaction with CLTX peptide or CLTX-CAR T cells was reminiscent of another study using CLTX-coated liposome (57). Together, our results suggested that membrane-bound MMP2 is necessary for CLTX-CAR T cell activation, while soluble MMP2 neither activates nor masks CLTX-CAR function.

\section{Discussion}

This study demonstrates that CLTX, a peptide component of scorpion venom, can be successfully incorporated into a CAR construct to redirect cytotoxic T cells to target GBMs. The antitumor effects of CLTX-CAR T cells were observed in multiple GBM xenograft models. We did not observe off-tumor targeting, and the CLTX-CAR T cells were well tolerated without evident systemic toxicity. Moreover, CLTX-Cy5.5 binding extended across a wider range of freshly-dissociated tumor cells and patientderived GBM cell lines than expression of antigens currently under consideration as CAR immunotherapy targets (i.e., IL13R $\alpha 2$, HER2 and EGFR). Our study also provides the first demonstration that a natural toxin peptide can be exploited to generate CAR T cells.

The criteria for selection of GBM-associated immunotherapy targets are particularly stringent because of the sensitivity of the brain to off-target activity or reactions to the therapy (3). For the target antigens currently under clinical investigation, one selection criterion is their specific expression on GBM tumors. IL13R $\alpha 2$ has negligible expression in normal brain and elsewhere (except as a cancer-testis antigen) (58, 59). EGFRvIII is the most common mutated form of EGFR restricted to multiple cancers including GBM $(60,61)$. Other antigen targets are over-expressed by tumor GBM cells as compared to normal cells (e.g. EGFR), and HER2, while moderately expressed in some normal tissues, shows only minimal expression 
on postnatal neurons or glial cells $(62,63)$. Initial development of CLTX-CAR T cells was justified by the demonstrated safety of CLTX itself in previous preclinical and clinical studies (28). Specifically, no toxicity was observed in a clinical study using CLTX to deliver ${ }^{131}$ I to tumor sites, or in preclinical studies leading to clinical use of fluorophore-conjugated CLTX as a real-time imaging agent during surgery (28, 29). Here we evaluated the potential toxicity of CLTX-CAR T cells in mouse models using multiple strategies, as CLTX binds to mouse glioma cells supporting its relevance for assessing off-tumor toxicities. First, intravenously delivered large-amount of CAR T cells showed no systemic toxicity (Fig. 5F). Further, the regional delivery of CAR T cells into tumor-bearing mice provided a more clinicallyrelevant condition where the CAR T cells can be activated by GBM tumors to assess off-tumor targeting (Fig. S5). Results of these preclinical studies in mice suggest that CLTX-CAR T cells mediate negligible off-tumor toxicities and provide support for its evaluation in GBM patients.

Tumor recurrence remains a barrier to successful immunotherapy for GBM. Recurrence is commonly associated with the presence of certain cell subpopulation(s), so-called glioma stem cells (GSCs) that are resistant to radiotherapy and chemotherapy, and are characterized by high tumor-initiating potential (39, $64,65)$. GSCs are usually resistant to conventional therapies, and a critical consideration of GBMtargeted immunotherapy has been the capability to eliminate GSCs $(39,66)$. Indeed, previous studies have demonstrated that GSCs can be as responsive to CAR T cell-mediated cytotoxicity as more differentiated GBM cells $(16,35,40)$, indicating that GSCs are potentially targetable by CAR T cell therapy despite their intrinsic immunosuppressive properties $(67,68)$. Consistent with this expectation, functional evaluations of CLTX-CAR T cells throughout this study have utilized patient-derived TS lines, which maintain stem-cell like properties under appropriate culture conditions (69). On the other hand, we were able to characterize GSCs from freshly-dispersed GBM samples by surface markers CD133 and CD44, representing different GBM molecular subtypes (65, 70, 71), and CLTX-Cy5.5 binding appeared to be similar in either GSC or non-GSC populations. All evidence suggests that CLTX-CAR T cells inherit the 
binding properties of CLTX-Cy5.5 conjugates, and thus will be active against both GSC and non-GSC populations in patient tumors, suggesting the potential to target the "seed” of GBM recurrence.

The choice of co-stimulatory signal has proven to be a critical element of CAR design. CAR T cells with CD28 $(72,73)$ or $4-1 \mathrm{BB}(8,74)$, costimulatory signals have been introduced to patients with hematological and solid tumors. In general, compared with CD28, 4-1BB co-stimulation has resulted in slower but more long-lasting $\mathrm{T}$ cell activation, as indicated by differential metabolic profiling (47), and consistent with CAR T cell expansion dynamics when co-infused into B-ALL patients (75). Surprisingly, in this study we found that CLTX-CAR T cells only displayed degranulation and killing antitumor responses when incorporating CD28 co-stimulation, while 4-1BB co-stimulation reduced both short-term and long-term effector activity for CLTX-CAR T cells. One possible explanation may be the nature of CLTX's interaction with its receptors. Although the binding affinity between CLTX and its receptor (membrane bound MMP-2 as shown in our study) to be necessary for CAR recognition is not known, an early study identified a low-affinity CLTX receptor which showed high abundance on GBM cell membranes (76). Since CD28 has been reported to lower the affinity threshold for TCR activation (77), it is possible that interaction between the CLTX tumor-recognition domain and low-affinity GBM receptors requires CD28 co-stimulation to sufficiently initiate CLTX-CAR T cell activation. We noted that along

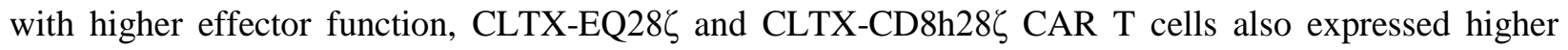
levels of exhaustion markers compared to the other CLTX-CARs evaluated. This observation could result from incomplete activation of the other non-CD28-bearing CLTX-CAR T cells upon tumor binding, as T cell exhaustion markers are induced upon antigen engagement and may also serve as indicators of T cell activation (78).

As notes, the composition of the CLTX receptor and its interaction with CLTX peptide is not well defined. The well-characterized inhibition of GBM cell migration and invasion by CLTX $(26,79)$ has been variously attributed to CLTX association with chloride channel CLCN3 and inhibiting the Cl- flux required for cell shape changes during invasion (27), or to CLTX inhibiting MMP2 thereby decreasing 
extracellular matrix cleavage (26). Interpretation of immunoprecipitation-based assays of interaction between CLTX and its receptor(s) has been complicated by difficulties distinguishing between direct interaction and indirect association. Here using genetic manipulations we observed that MMP2 expression is required for CLTX-CAR T cell activation, consistent with MMP2 serving as the CLTX receptor or a critical component within a receptor complex. Importantly, CLTX-CAR T cells did not respond to secreted MMP2, thus preserving their activation potential even in a soluble MMP2-rich microenvironment (80). The development of CLTX-CAR T cells provide evidence that CAR T cells are able to selectively target membrane-bound form of secreted enzymes, in addition to the previouslyreported CAR designs against soluble proteins $(81,82)$. Further, since CAR T cell activation requires direct antigen engagement, our results are consistent with MMP2 being a direct target for CLTX, with their interaction recruiting other associated proteins such as CLCN3 (Fig. 6I and S6H). Similar pattern of CLTX's association with GBM cells was also suggested by previous studies using CLTX-coated nanoparticles and fusion proteins $(57,83)$. Of note, correlation was observed between MMP2 expression, degranulation (Fig. 6B), in vivo CLTX-CAR efficacy (Fig. 6F), and CLTX-Cy5.5 staining intensity (Fig. 6C). Our result suggest that CLTX-Cy5.5 staining of GBM cells could serve as an efficient strategy for identifying the potential responsiveness of GBM tumors to CLTX-CAR T cell therapy.

A generalizable finding arising from our in vivo CLTX-CAR studies is the observation that antitumor function of GBM-targeted CARs may be inhibited by adaptive resistance mechanisms of GBM tumors (Fig. 4). Although most of the patient-derived GBM cell lines used in this study did not express PD-L1 (data not shown), induction of PD-L1 expression by GBM xenografts, as well as TS lines, did occur after CAR therapy, and seemed to depend on IFN- $\gamma$ receptor expression, consistent with the mechanism characterized in metastatic melanomas (51). The inhibitory effect of PD-L1 to CLTX-CAR T cells acts mainly on the long-term in vivo function, as initial CLTX-CAR activation (degranulation) was not well correlated with the IFN $\gamma$ RA (CD119) expression potentially linked to checkpoint inhibition (Fig. S6D). Our results lead to the potential of correlating tumor IFN- $\gamma$ receptor expression with CLTX-CAR T cell 
therapeutic effect, as well as the possibility of combining CLTX-CAR T cells with checkpoint inhibitors for more effective GBM clearance.

Overall, we were able to observe broad GBM-targeting capability of CLTX-CAR T cells, consistent with the wide expression of its receptor, MMP2, in GBM samples (84). Of particular importance, CLTX-CAR $\mathrm{T}$ cells elicited potent immune responses against tumor cells with no or little expression of other targetable antigens (IL13R $\alpha 2$, HER2 and EGFR). Through its combination of selectivity and ubiquity, CLTX-CAR T cells address two major hurdles to effective immunotherapy treatment of GBM: reduction of antigen escape while maintaining tumor cell restriction. We suggest that CLTX-CAR T cells are a candidate for clinical development of anti-GBM immunotherapy, circumventing antigen escape either as a single agent, or in combination with other CAR T cells or immunotherapeutic strategies.

\section{Materials and Methods}

\section{Study design}

In this study we evaluated the antitumor potency of CLTX-CAR T cells against GBMs. First, CLTX binding was verified in various GBM samples. All freshly dispersed tumors were processed into single cells following the laboratory's standard protocol, and PBT-TS lines were generated from freshly dispersed tumor cells and maintained at the standard and well-established condition using neurosphere media. For the experiments on functional evaluation, CAR T cells were generated from three different healthy donors, and tested against multiple GBM models established by TS lines. For in vitro assays, 2-5 replicates within each condition were used to sufficiently represent intra-group variations and allow for defining statistical significance. In all in vivo experiments, 6-8 week-old NOD/SCID/IL2R-/- (NSG) mice were used, and 4-8 mice were included within each group which enabled us to statistically distinguish tumor sizes and survival rates across groups. Before CAR T cell treatment, mice were grouped with similar average tumor sizes across groups. The health condition of mice was monitored at a daily basis by the Department of Comparative Medicine at City of Hope, with euthanasia applied according to the 
American Veterinary Medical Association Guidelines. For every single mouse euthanized, brain was collected to confirm the presence of GBM tumors. The pathological conditions of mouse organs were determined by a mouse pathologist from the Veterinary Pathology Program at City of Hope..

\section{Generation of CLTX-conjugated peptides}

Conjugation of CLTX (Alomone Labs) with Cy5.5 fluorescent dye (GE Healthcare) or biotin (Thermo Fisher Scientific) was performed by the Synthetic and Biopolymer Chemistry Core at City of Hope.

Isolation of primary brain tumor cells, establishment of neurospheres and other cell lines

Primary brain tumor (PBT) cells were obtained from GBM patient resections at COH under protocols approved by the $\mathrm{COH}$ Internal Review Board. Resected brain tumor specimens were digested using a human tumor dissociation kit (Miltenyi Biotech Inc) to generate PBT cells. TS lines were subsequently established from PBTs and maintained as described previously $(16,37)$. To generate cells for in vivo biophotonic imaging, these cells were engineered to express the ffLuc reporter gene as previously described (16). Differentiation of TS lines was performed by withdraw of EGF/FGF in the TS culture media and supplement with $10 \%$ fetal calf serum (FCS), as depicted previously (16). FB-NSCs were established and characterized as reported in previous studies (52-54). Astrocytes and NPCs are differentiated from health donor-derived iPSCs based on established protocols $(85,86)$.

\section{DNA constructs}

All CLTX-CAR constructs contain a CLTX peptide and the cytoplasmic domain of human CD3 zeta, with different spacers including: IgG4EQ [IgG4 with two point mutations (L235E, N297Q) (7)]; $\Delta$ CH2: IgG4-Fc with the CH2-domain deleted; CD8h: CD8 hinge; L: a synthetic 10 amino acids short linker. CAR constructs also contain CD4 or CD28 transmembrane domains, and CD28 or 4-1BB costimulatory domains. All domains have been previously reported (43, 45, 87). A truncated CD19 was also introduced 
in the construct to allow for potential enrichment and tracking of transduced cells. The firefly luciferase (ffLuc)-GFP construct for tumor biophotonic imaging was generated as described previously (16).

\section{CAR T cell production}

Blood products were obtained from healthy donors under protocols approved by the City of Hope (COH) Internal Review Board, and naïve/memory $\mathrm{T}$ cell $(\mathrm{Tn} / \mathrm{mem})$ isolation followed the procedures described in previous studies $(87,88)$. In brief, peripheral blood mononuclear cells (PBMCs) were isolated by density gradient centrifugation over Ficoll-Paque (GE Healthcare) and then underwent sequential rounds of CliniMACS ${ }^{\circledR} /$ AutoMACS ${ }^{\circledR}$ depletion to remove CD14, CD25-expressing cells, followed by a CD62L positive selection for Tn/mem cells. To generate CAR T cell products, T cells were stimulated with Dynabeads ${ }^{\circledR}$ Human T expander CD3/CD28 (Invitrogen) at a 1:3 ratio (T cell:bead), and transduced with lentivirus to express CAR (MOI=2) in X-VIVO 15 (Lonza) containing 10\% FCS with $5 \mu \mathrm{g} / \mathrm{mL}$ protamine sulfate (APP Pharmaceuticals), $50 \mathrm{U} / \mathrm{mL}$ rhIL-2 and $0.5 \mathrm{ng} / \mathrm{mL}$ rhIL-15. Cultures were then maintained at $37^{\circ} \mathrm{C}, 5 \% \mathrm{CO}_{2}$ under the same condition of media and cytokines (cytokines were supplied every other day). On day 7 post transduction, the CD3/CD28 Dynabeads were removed from cultures using the DynaMag-50 magnet (Invitrogen). CAR-transduced $\mathrm{T}$ cells were enriched by positive selections using anti-CD19 magnetic beads (Stem Cell Technologies). Cultures were propagated for 14-16 days before applying to assays or get cryo-preserved. Mock-transduced T cells were generated by stimulating and culturing Tn/mem cells from the same donors as described above, without lentivirus addition.

\section{Flow cytometry}

Cells were harvested and stained as described previously (7). T cell phenotype was detected using fluorochrome-conjugated antibodies against CD3, CD4, CD8 and CD45 (BD Biosciences). Transgene expression was determined by staining for the truncated CD19 (BD Biosciences), and all engineered T cells were gated as the $\mathrm{CD}^{+}$, $\mathrm{CD} 45^{+}, \mathrm{CD} 19^{+}$population unless specifically mentioned in figure legends. $\mathrm{T}$ cell activation was determined using antibodies against CD69, CD107a, CD137, IFN- $\gamma$ (BD 
Biosciences) and PD-1 (BioLegend). Tumor cells were stained with Cy5.5-CLTX, or antibodies against IL13Ra2, HER2, EGFR (BioLegend), CD44 (BD Biosciences) and CD133 (Miltenyi Biotech Inc.). All samples were analyzed via a Macsquant Analyzer (Miltenyi Biotec Inc.) and processed via FlowJo v10.

In vitro T cell functional assays

To acquire GBM cells for testing CLTX-CAR T cytotoxicity and activity, TSs were dissociated with cold Accutase (Innovative Cell Tec) and resuspended in DMEM:F12 (Irvine Scientific) medium supplied with 10\% FCS. CLTX-CAR T cells were then washed and resuspended in the same medium, and added to the PBT cells. To test for degranulation, CLTX-CAR T cells were incubated with GBM cells (E:T=1:1) for 5 $\mathrm{h}$ in the presence of CD107a antibody and Golgistop ${ }^{\mathrm{TM}}$ protein transport inhibitor (BD Biosciences). To test for CAR T cell killing potency, CLTX-CAR T cells were co-cultured with GBM cells at different E:T ratios for 24-72 h as indicated in individual legends. For the repetitive tumor challenge assay, 4,000 CLTX-CAR T cells were initially co-cultured with 16,000 GBM cells, and then rechallenged with additional 32,000 GBM cells every 48 h for 3 rounds (48). Killing was quantified by analyzing viable tumor cells (CD45 DAPI') after co-culture by flow cytometry.

\section{Immunofluorescence staining}

To visualize immunological-synapses, GBM cells were harvested from TS lines as described above, and plated for $12 \mathrm{~h}$ to adhere. CLTX-CAR T cells were then added to GBM cells and incubated for 3h before fixation with 4\% paraformaldehyde (in PBS). Fixed cells were first stained with following antibodies: rabbit-anti-human pCD3ৎ (Abcam, EP776(2)Y), rabbit-anti-human MMP2 (Abcam, EPR1184) and mouse-anti-human CLCN3 (Abcam, N258/5). Permeabilization and cytoskeleton staining was then performed using an F-actin Visualization Biochem Kit (Cytoskeleton Inc.). To stain for GBM-associated antigens, mouse brains bearing GBM xenografts were harvested, embedded into paraffin and sectioned as described previously (16). IF staining was performed on paraffin sections using the procedures of a previous study (89), with the following antibodies: goat-anti-human IL13R $\alpha 2$ (R\&D systems, polyclonal), 
mouse-anti-human EGFR (Dako, DAK-H1-WT), and CLTX-biotin. All slides for immunofluorescence assays were observed with an LSM Airyscan 880 (Zeiss) and images were processed with Zen (Zeiss). Quantification of immunofluoresence images was performed using Image Pro (Media Cybernetics).

GBM xenograft studies

All mouse experiments were approved by the $\mathrm{COH}$ Institutional Animal Care and Use Committee. Orthotopic GBM models were generated using NSG mice as previously described (50). Briefly, on day 0, ffLuc $^{+}$GBM cells $\left(1 \times 10^{5}\right)$ were stereotactically implanted into the right forebrain. After 8 days, mice were then treated intracranially with $0.5 \times 10^{6} \mathrm{CAR} \mathrm{T}$ cells. Tumor volumes were determined by in vivo non-invasive optical biophotonic imaging using a Xenogen IVIS 100 as previously described (16). For subcutaneous tumor xenografts, GBM cells $\left(5 \times 10^{6}\right)$ were injected into the left flank of NSG mice and tumors were allowed to grow for 7-14 days. CAR T cells $\left(2 \times 10^{6}\right)$ were injected intratumorally and tumors sizes were monitored until animal euthanasia when tumor sizes reached $15 \mathrm{~mm} \times 15 \mathrm{~mm}$. To acquire single cells for flow cytometric analysis, xenograft tumors were cut into pieces, physically dissociated and filtered. The pathological conditions of mouse organs were determined by the Veterinary Pathology Program at City of Hope.

\section{Immunohistochemistry}

Mouse brain harvesting, sectioning and immunohistochemistry (IHC) assays were performed as described previously (16). Antibodies used in IHC assays include: mouse-anti-human CD3 (Dako, F7.2.38), rabbitanti-human Granzyme B (eBiosciences, 496B), rabbit-anti-human-PDL1 (Cell Signaling Technology, E1L3N). The slides for IHC assays were scanned via a NanoZoomer 2.0-HT Digital slide scanner (Hamamatsu) and processed with NDP.view2 (Hamamatsu).

Quantitative real-time PCR 
Total mRNA from tumor cells was isolated by RNeasy Mini Kit (Qiagen Inc.). cDNA was then synthesized using an Omniscript RT Kit (Qiagen Inc.). Quantitative real-time PCR was performed using SYBR Green PCR Master mix (Applied Biosystems) in a ViiA ${ }^{\mathrm{TM}}-7$ RT-PCR system (Thermo Fisher Scientific). Primer sequences are available upon request. The comparative Ct values of genes of interest were normalized to the $\mathrm{Ct}$ value of $\beta$-actin. Then, the $2^{-\Delta c t}$ method was used to determine the relative expression of the genes, while the $2^{-\Delta \Delta c t}$ method was used to calculate the fold changes of gene expression over control.

\section{Statistics}

Data analysis was performed using Prism v6.0 (GraphPad Software) and presented as stated in individual figure legends. Comparisons were determined using Student's t-test (two groups) or one-way ANOVA (three or more groups). For comparisons between three or more groups, Bonferroni's Multiple Comparison Tests were used to compare all or selected pairs of data (95\% confidence intervals). Comparison of Kaplan-Meier survival data was performed using the Log-rank (Mantel-Cox) test. Detailed comparisons in each experiment are described in figure legends.

\section{Study Approval}

All mouse experiments were approved by the City of Hope Institute Animal Care and Use Committee, Duarte, CA. Use of all human subjects materials (human CAR T cell production and patient-derived GBM spheres) was approved by the City of Hope Institutional Review Board, Duarte, CA.

\section{Supplementary Materials}

Materials and Methods

Fig. S1. Antigen expression on PBT-TS lines.

Fig. S2. Activation of CLTX-CAR T cells after GBM stimulation. 


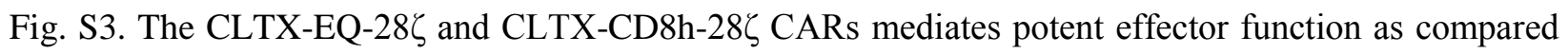
with other CLTX-CAR constructs.

Fig. S4. CLTX-CAR T cells target GBM xenografts.

Fig. S5. CLTX-CAR T cells do not exhibit off-tumor targeting in tumor-bearing mice.

Fig. S6. MMP2 is necessary for CLTX-CAR T cell activation.

Video.S1. Killing of PBT003-4-TS-derived GBMs during a 72h co-culture with the mixture of IL13Ra2-, HER2- and EGFRvIII-targeted CAR T cells

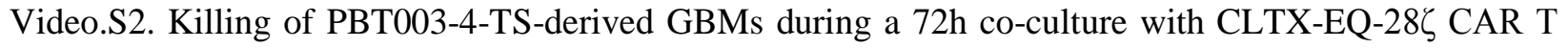
cells

\section{References}

1. E. G. Van Meir et al., Exciting new advances in neuro-oncology: the avenue to a cure for malignant glioma. CA: a cancer journal for clinicians 60, 166 (May-Jun, 2010).

2. B. M. Alexander, T. F. Cloughesy, Adult Glioblastoma. Journal of clinical oncology : official journal of the American Society of Clinical Oncology 35, 2402 (Jul 20, 2017).

3. J. H. Sampson, M. V. Maus, C. H. June, Immunotherapy for Brain Tumors. Journal of Clinical Oncology 35, 2450 (Jul 20, 2017).

4. B. D. Choi, W. T. Curry, B. S. Carter, M. V. Maus, Chimeric antigen receptor T-cell immunotherapy for glioblastoma: practical insights for neurosurgeons. Neurosurg Focus 44, (Jun, 2018).

5. S. J. Priceman, S. J. Forman, C. E. Brown, Smart CARs engineered for cancer immunotherapy. Current opinion in oncology 27, 466 (Nov, 2015).

6. A. D. Fesnak, C. H. June, B. L. Levine, Engineered T cells: the promise and challenges of cancer immunotherapy. Nature reviews. Cancer 16, 566 (Aug 23, 2016). 
7. M. Jonnalagadda et al., Chimeric antigen receptors with mutated IgG4 Fc spacer avoid fc receptor binding and improve T cell persistence and antitumor efficacy. Molecular therapy : the journal of the American Society of Gene Therapy 23, 757 (Apr, 2015).

8. C. E. Brown et al., Regression of Glioblastoma after Chimeric Antigen Receptor T-Cell Therapy. The New England journal of medicine 375, 2561 (Dec 29, 2016).

9. N. Ahmed et al., HER2-Specific Chimeric Antigen Receptor-Modified Virus-Specific T Cells for Progressive Glioblastoma: A Phase 1 Dose-Escalation Trial. JAMA oncology 3, 1094 (Aug 01, 2017).

10. D. M. O'Rourke et al., A single dose of peripherally infused EGFRvIII-directed CAR T cells mediates antigen loss and induces adaptive resistance in patients with recurrent glioblastoma. Science translational medicine 9, (Jul 19, 2017).

11. I. Scarfo, M. V. Maus, Current approaches to increase CAR T cell potency in solid tumors: targeting the tumor microenvironment. Journal for immunotherapy of cancer 5, 28 (2017).

12. S. Gill, C. H. June, Going viral: chimeric antigen receptor T-cell therapy for hematological malignancies. Immunological reviews 263, 68 (Jan, 2015).

13. R. G. Verhaak et al., Integrated genomic analysis identifies clinically relevant subtypes of glioblastoma characterized by abnormalities in PDGFRA, IDH1, EGFR, and NF1. Cancer cell 17, 98 (Jan 19, 2010).

14. A. P. Patel et al., Single-cell RNA-seq highlights intratumoral heterogeneity in primary glioblastoma. Science 344, 1396 (Jun 20, 2014).

15. C. E. Brown et al., Glioma IL13Ralpha2 is associated with mesenchymal signature gene expression and poor patient prognosis. PloS one 8, e77769 (2013).

16. C. E. Brown et al., Stem-like tumor-initiating cells isolated from IL13Ralpha2 expressing gliomas are targeted and killed by IL13-zetakine-redirected T Cells. Clinical cancer research : an official journal of the American Association for Cancer Research 18, 2199 (Apr 15, 2012). 
17. C. E. Brown et al., Bioactivity and Safety of IL13Ralpha2-Redirected Chimeric Antigen Receptor CD8+ T Cells in Patients with Recurrent Glioblastoma. Clinical cancer research : an official journal of the American Association for Cancer Research 21, 4062 (Sep 15, 2015).

18. J. H. Sampson et al., Immunologic escape after prolonged progression-free survival with epidermal growth factor receptor variant III peptide vaccination in patients with newly diagnosed glioblastoma. Journal of clinical oncology : official journal of the American Society of Clinical Oncology 28, 4722 (Nov 1, 2010).

19. Q. Wang et al., Tumor Evolution of Glioma-Intrinsic Gene Expression Subtypes Associates with Immunological Changes in the Microenvironment. Cancer cell 32, 42 (Jul 10, 2017).

20. S. A. Grupp et al., Chimeric antigen receptor-modified T cells for acute lymphoid leukemia. The New England journal of medicine 368, 1509 (Apr 18, 2013).

21. D. L. Porter, B. L. Levine, M. Kalos, A. Bagg, C. H. June, Chimeric antigen receptor-modified T cells in chronic lymphoid leukemia. The New England journal of medicine 365, 725 (Aug 25, 2011).

22. R. J. Brentjens et al., CD19-targeted T cells rapidly induce molecular remissions in adults with chemotherapy-refractory acute lymphoblastic leukemia. Science translational medicine 5, 177ra38 (Mar 20, 2013).

23. E. Rajesh, L. S. Sankari, L. Malathi, J. R. Krupaa, Naturally occurring products in cancer therapy. Journal of pharmacy \& bioallied sciences 7, S181 (Apr, 2015).

24. J. A. DeBin, J. E. Maggio, G. R. Strichartz, Purification and characterization of chlorotoxin, a chloride channel ligand from the venom of the scorpion. The American journal of physiology 264, C361 (Feb, 1993).

25. L. Soroceanu, Y. Gillespie, M. B. Khazaeli, H. Sontheimer, Use of chlorotoxin for targeting of primary brain tumors. Cancer research 58, 4871 (Nov 1, 1998).

26. J. Deshane, C. C. Garner, H. Sontheimer, Chlorotoxin inhibits glioma cell invasion via matrix metalloproteinase-2. The Journal of biological chemistry 278, 4135 (Feb 7, 2003). 
27. M. B. McFerrin, H. Sontheimer, A role for ion channels in glioma cell invasion. Neuron glia biology 2, 39 (Feb, 2006).

28. A. N. Mamelak et al., Phase I single-dose study of intracavitary-administered iodine-131-TM-601 in adults with recurrent high-grade glioma. Journal of clinical oncology : official journal of the American Society of Clinical Oncology 24, 3644 (Aug 1, 2006).

29. J. Parrish-Novak et al., Nonclinical Profile of BLZ-100, a Tumor-Targeting Fluorescent Imaging Agent. International journal of toxicology 36, 104 (Mar/Apr, 2017).

30. L. Zhao, X. Shi, J. Zhao, Chlorotoxin-conjugated nanoparticles for targeted imaging and therapy of glioma. Current topics in medicinal chemistry 15, 1196 (2015).

31. O. Veiseh et al., Chlorotoxin bound magnetic nanovector tailored for cancer cell targeting, imaging, and siRNA delivery. Biomaterials 31, 8032 (Nov, 2010).

32. M. Veiseh et al., Tumor paint: a chlorotoxin:Cy5.5 bioconjugate for intraoperative visualization of cancer foci. Cancer research 67, 6882 (Jul 15, 2007).

33. J. Fidel et al., Preclinical Validation of the Utility of BLZ-100 in Providing Fluorescence Contrast for Imaging Spontaneous Solid Tumors. Cancer research 75, 4283 (Oct 15, 2015).

34. S. A. Lyons, J. O'Neal, H. Sontheimer, Chlorotoxin, a scorpion-derived peptide, specifically binds to gliomas and tumors of neuroectodermal origin. Glia 39, 162 (Aug, 2002).

35. N. Ahmed et al., HER2-specific $\mathrm{T}$ cells target primary glioblastoma stem cells and induce regression of autologous experimental tumors. Clinical cancer research : an official journal of the American Association for Cancer Research 16, 474 (Jan 15, 2010).

36. J. Lee et al., Tumor stem cells derived from glioblastomas cultured in bFGF and EGF more closely mirror the phenotype and genotype of primary tumors than do serum-cultured cell lines. Cancer cell 9, 391 (May, 2006).

37. C. E. Brown et al., Recognition and killing of brain tumor stem-like initiating cells by CD8+ cytolytic T cells. Cancer research 69, 8886 (Dec 1, 2009). 
38. H. S. Gunther et al., Glioblastoma-derived stem cell-enriched cultures form distinct subgroups according to molecular and phenotypic criteria. Oncogene 27, 2897 (May 1, 2008).

39. J. D. Lathia, S. C. Mack, E. E. Mulkearns-Hubert, C. L. Valentim, J. N. Rich, Cancer stem cells in glioblastoma. Genes \& development 29, 1203 (Jun 15, 2015).

40. R. A. Morgan et al., Recognition of glioma stem cells by genetically modified T cells targeting EGFRvIII and development of adoptive cell therapy for glioma. Human gene therapy 23, 1043 (Oct, 2012).

41. S. K. Singh et al., Identification of human brain tumour initiating cells. Nature 432, 396 (Nov 18, 2004).

42. A. Pietras et al., Osteopontin-CD44 signaling in the glioma perivascular niche enhances cancer stem cell phenotypes and promotes aggressive tumor growth. Cell stem cell 14, 357 (Mar 6, 2014).

43. X. Wang et al., Phase 1 studies of central memory-derived CD19 CAR T-cell therapy following autologous HSCT in patients with B-cell NHL. Blood 127, 2980 (Jun 16, 2016).

44. M. Hudecek et al., The Non-Signaling Extracellular Spacer Domain of CD19-Specific Chimeric Antigen Receptors Is Decisive for in Vivo Anti-Tumor Activity. Blood 120, (Nov 16, 2012).

45. S. J. Priceman et al., Co-stimulatory signaling determines tumor antigen sensitivity and persistence of CAR T cells targeting PSCA+ metastatic prostate cancer. Oncoimmunology 7, e1380764 (2018)

46. A. H. Long et al., 4-1BB costimulation ameliorates $\mathrm{T}$ cell exhaustion induced by tonic signaling of chimeric antigen receptors. Nature medicine 21, 581 (Jun, 2015).

47. O. U. Kawalekar et al., Distinct Signaling of Coreceptors Regulates Specific Metabolism Pathways and Impacts Memory Development in CAR T Cells. Immunity 44, 380 (Feb 16, 2016).

48. D. Wang et al., Glioblastoma-targeted CD4+ CAR T cells mediate superior antitumor activity. JCI insight 3, (May 17, 2018).

49. J. Eyquem et al., Targeting a CAR to the TRAC locus with CRISPR/Cas9 enhances tumour rejection. Nature 543, 113 (Mar 02, 2017). 
50. K. S. Kahlon et al., Specific recognition and killing of glioblastoma multiforme by interleukin 13zetakine redirected cytolytic T cells. Cancer research 64, 9160 (Dec 15, 2004).

51. A. Garcia-Diaz et al., Interferon Receptor Signaling Pathways Regulating PD-L1 and PD-L2 Expression. Cell reports 19, 1189 (May 9, 2017).

52. R. C. Rockne et al., Long-term stability and computational analysis of migration patterns of LMYC immortalized neural stem cells in the brain. PloS one 13, e0199967 (2018).

53. Z. Li et al., L-MYC Expression Maintains Self-Renewal and Prolongs Multipotency of Primary Human Neural Stem Cells. Stem cell reports 7, 483 (Sep 13, 2016).

54. M. Nakagawa, N. Takizawa, M. Narita, T. Ichisaka, S. Yamanaka, Promotion of direct reprogramming by transformation-deficient Myc. Proceedings of the National Academy of Sciences of the United States of America 107, 14152 (Aug 10, 2010).

55. L. Tatenhorst, U. Rescher, V. Gerke, W. Paulus, Knockdown of annexin 2 decreases migration of human glioma cells in vitro. Neuropathology and applied neurobiology 32, 271 (Jun, 2006).

56. M. Egeblad, Z. Werb, New functions for the matrix metalloproteinases in cancer progression. Nature reviews. Cancer 2, 161 (Mar, 2002).

57. C. Qin et al., The impact of a chlorotoxin-modified liposome system on receptor MMP-2 and the receptor-associated protein ClC-3. Biomaterials 35, 5908 (Jul, 2014).

58. W. Debinski, D. M. Gibo, S. W. Hulet, J. R. Connor, G. Y. Gillespie, Receptor for interleukin 13 is a marker and therapeutic target for human high-grade gliomas. Clinical Cancer Research 5, 985 (May, 1999).

59. J. S. Jarboe, K. R. Johnson, Y. Choi, R. R. Lonser, J. K. Park, Expression of interleukin-13 receptor alpha 2 in glioblastoma multiforme: Implications for targeted therapies. Cancer research 67, 7983 (Sep 1, 2007).

60. G. Li, A. J. Wong, EGF receptor variant III as a target antigen for tumor immunotherapy. Expert Rev Vaccines 7, 977 (Sep, 2008). 
61. E. Padfield, H. P. Ellis, K. M. Kurian, Current Therapeutic Advances Targeting EGFR and EGFRvIII in Glioblastoma. Frontiers in oncology 5, 5 (2015).

62. J. G. Zhang et al., Antigenic profiling of glioma cells to generate allogeneic vaccines or dendritic cell-based therapeutics. Clinical cancer research : an official journal of the American Association for Cancer Research 13, 566 (Jan 15, 2007).

63. J. G. Zhang et al., Tumor antigen precursor protein profiles of adult and pediatric brain tumors identify potential targets for immunotherapy. Journal of neuro-oncology 88, 65 (May, 2008).

64. S. Bao et al., Glioma stem cells promote radioresistance by preferential activation of the DNA damage response. Nature 444, 756 (Dec 7, 2006).

65. X. Jin et al., Targeting glioma stem cells through combined BMI1 and EZH2 inhibition. Nature medicine 23, 1352 (Nov, 2017).

66. J. Chen, R. M. McKay, L. F. Parada, Malignant glioma: lessons from genomics, mouse models, and stem cells. Cell 149, 36 (Mar 30, 2012).

67. J. Wei et al., Glioblastoma cancer-initiating cells inhibit T-cell proliferation and effector responses by the signal transducers and activators of transcription 3 pathway. Molecular cancer therapeutics 9, 67 (Jan, 2010).

68. T. Di Tomaso et al., Immunobiological characterization of cancer stem cells isolated from glioblastoma patients. Clinical cancer research : an official journal of the American Association for Cancer Research 16, 800 (Feb 1, 2010).

69. H. D. Hemmati et al., Cancerous stem cells can arise from pediatric brain tumors. Proceedings of the National Academy of Sciences of the United States of America 100, 15178 (Dec 9, 2003).

70. K. P. L. Bhat et al., Mesenchymal differentiation mediated by NF-kappaB promotes radiation resistance in glioblastoma. Cancer cell 24, 331 (Sep 9, 2013).

71. D. V. Brown et al., Coexpression analysis of CD133 and CD44 identifies Proneural and Mesenchymal subtypes of glioblastoma multiforme. Oncotarget 6, 6267 (Mar 20, 2015). 
72. D. W. Lee et al., T cells expressing CD19 chimeric antigen receptors for acute lymphoblastic leukaemia in children and young adults: a phase 1 dose-escalation trial. Lancet 385, 517 (Feb 7, 2015).

73. S. S. Neelapu et al., Axicabtagene Ciloleucel CAR T-Cell Therapy in Refractory Large B-Cell Lymphoma. The New England journal of medicine 377, 2531 (Dec 28, 2017).

74. D. L. Porter et al., Chimeric antigen receptor T cells persist and induce sustained remissions in relapsed refractory chronic lymphocytic leukemia. Science translational medicine 7, 303ra139 (Sep 02, 2015).

75. Z. Cheng et al., In Vivo Expansion and Antitumor Activity of Coinfused CD28- and 4-1BBEngineered CAR-T Cells in Patients with B Cell Leukemia. Molecular therapy : the journal of the American Society of Gene Therapy 26, 976 (Apr 4, 2018).

76. C. Geldres et al., T lymphocytes redirected against the chondroitin sulfate proteoglycan-4 control the growth of multiple solid tumors both in vitro and in vivo. Clinical cancer research : an official journal of the American Association for Cancer Research 20, 962 (Feb 15, 2014).

77. J. A. Punt, B. A. Osborne, Y. Takahama, S. O. Sharrow, A. Singer, Negative selection of CD4+CD8+ thymocytes by $\mathrm{T}$ cell receptor-induced apoptosis requires a costimulatory signal that can be provided by CD28. The Journal of experimental medicine 179, 709 (Feb 1, 1994).

78. A. C. Anderson, N. Joller, V. K. Kuchroo, Lag-3, Tim-3, and TIGIT: Co-inhibitory Receptors with Specialized Functions in Immune Regulation. Immunity 44, 989 (May 17, 2016).

79. L. Soroceanu, T. J. Manning, H. Sontheimer, Modulation of glioma cell migration and invasion using Cl- and K+ ion channel blockers. J Neurosci 19, 5942 (Jul 15, 1999).

80. M. Egeblad, Z. Werb, New functions for the matrix metalloproteinases in cancer progression. Nature Reviews Cancer 2, 161 (Mar, 2002).

81. Z. L. Chang et al., Rewiring T-cell responses to soluble factors with chimeric antigen receptors. Nature chemical biology 14, 317 (Mar, 2018). 
82. A. J. Hou, Z. L. Chang, M. H. Lorenzini, E. Zah, Y. Y. Chen, TGF-beta-responsive CAR-T cells promote anti-tumor immune function. Bioengineering \& translational medicine 3, 75 (May, 2018).

83. S. El-Ghlban et al., Chlorotoxin-Fc fusion inhibits release of MMP-2 from pancreatic cancer cells. BioMed research international 2014, 152659 (2014).

84. R. Beliveau et al., Expression of matrix metalloproteinases and their inhibitors in human brain tumors. Ann Ny Acad Sci 886, 236 (1999).

85. L. Li et al., GFAP Mutations in Astrocytes Impair Oligodendrocyte Progenitor Proliferation and Myelination in an hiPSC Model of Alexander Disease. Cell stem cell 23, 239 (Aug 2, 2018).

86. R. Krencik, S. C. Zhang, Directed differentiation of functional astroglial subtypes from human pluripotent stem cells. Nature protocols 6, 1710 (Oct 13, 2011).

87. C. E. Brown et al., Optimization of IL13Ralpha2-Targeted Chimeric Antigen Receptor T Cells for Improved Anti-tumor Efficacy against Glioblastoma. Molecular therapy : the journal of the American Society of Gene Therapy, (Oct 05, 2017).

88. X. Wang et al., Phenotypic and functional attributes of lentivirus-modified CD19-specific human CD8+ central memory T cells manufactured at clinical scale. Journal of immunotherapy 35, 689 (Nov-Dec, 2012).

89. R. Sugimura et al., Noncanonical Wnt signaling maintains hematopoietic stem cells in the niche. Cell 150, 351 (Jul 20, 2012).

Acknowledgements: We thank the Department of Comparative Medicine, and the cores of Synthetic and Biopolymer Chemistry, Small Animal Imaging, Light Microscopy, Mouse Pathology, Solid Tumor Pathology; as well as Brenda Chang, Juan Ruiz-Delgado and Dr. Lihong Weng for their technical assistance. We thank Dr. James M. Olson for interactive discussions and intellectual feedback on this work. Funding: This work was supported by grants from the Ben and Catherine Ivy Foundation and NIH 
grant P30CA33572 (cores). D.W. is supported by NCI fellowship 1F99CA234923-01. Author contributions: Designing research studies: D.W., S.J.F., M.E.B. and C.E.B.; Conducting experiments: D.W., R.S., W.C.C., B.A., S.W., X.Y., A.B., and A.S.; Data acquisition: D.W., R.S. and B.A.; Analysis and interpretation: D.W., D.A., M.E.B. and C.E.B.; Resources: M.G., K.A., Y.S., B.B., C.E.B. and S.J.F.; Writing manuscript: D.W., D.A., J.R.O., M.E.B. and C.E.B.; Supervision: S.J.F., M.E.B. and C.E.B. Competing interests: S.J.F. and C.E.B. receive royalty payments from Mustang Bio; all other authors declare no competing interests. Data and materials availability: PBT-TS lines are available from C.E.B. under a material transfer agreement with City of Hope. 
A
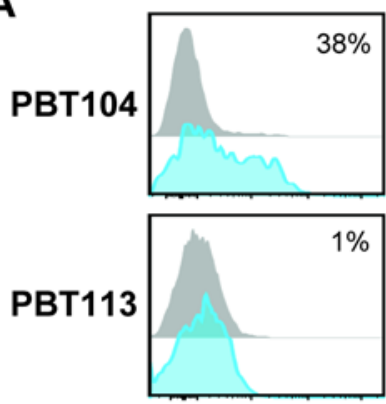

IL13R $\alpha 2$
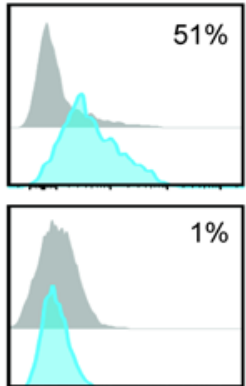

HER2
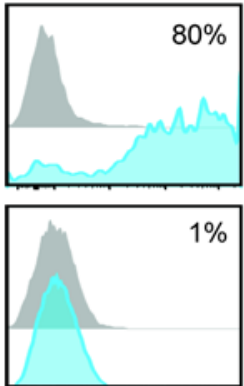

EGFR
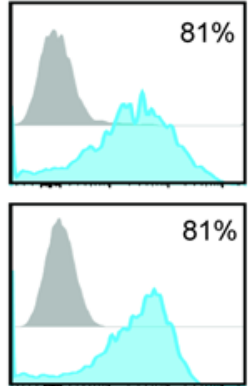

CLTX

B

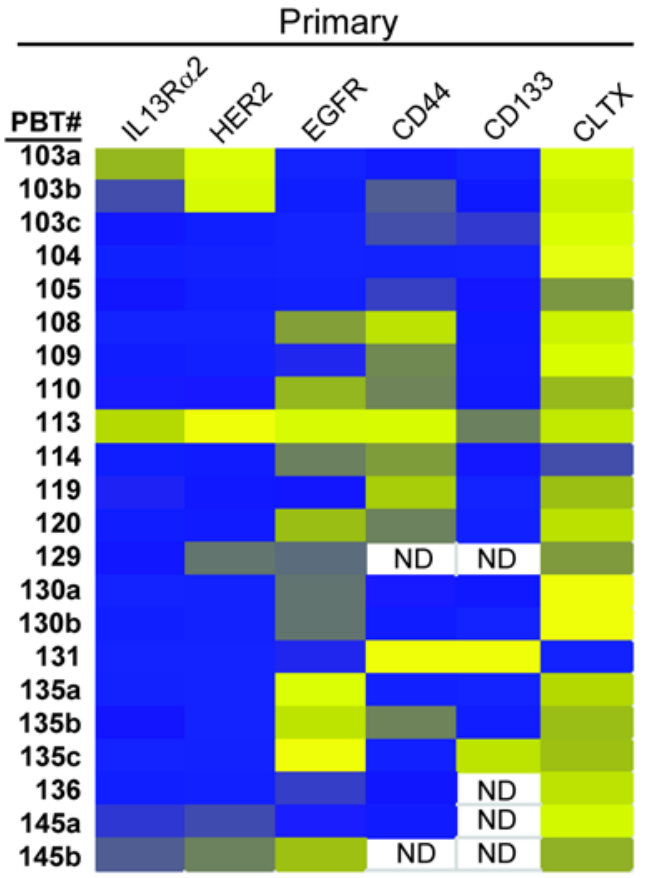

D

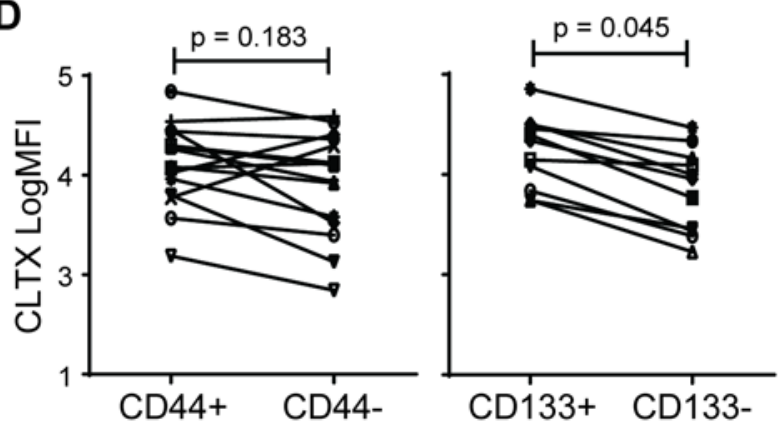

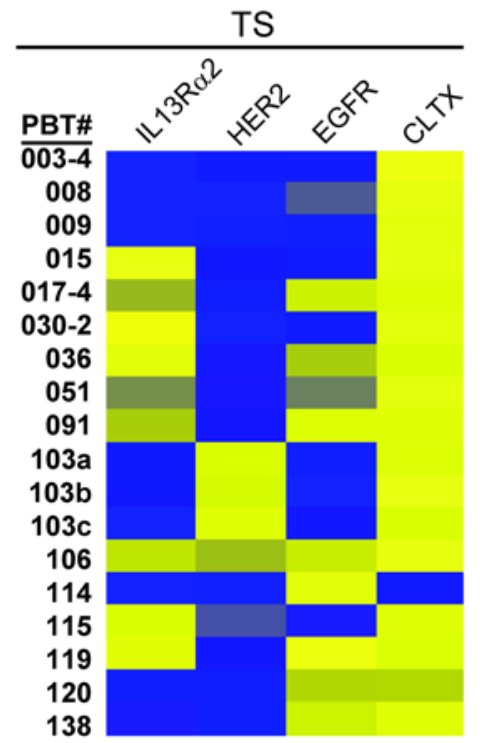

Staining:

$0 \%$ $100 \%$

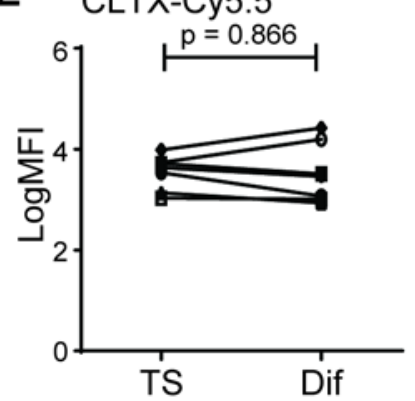

C
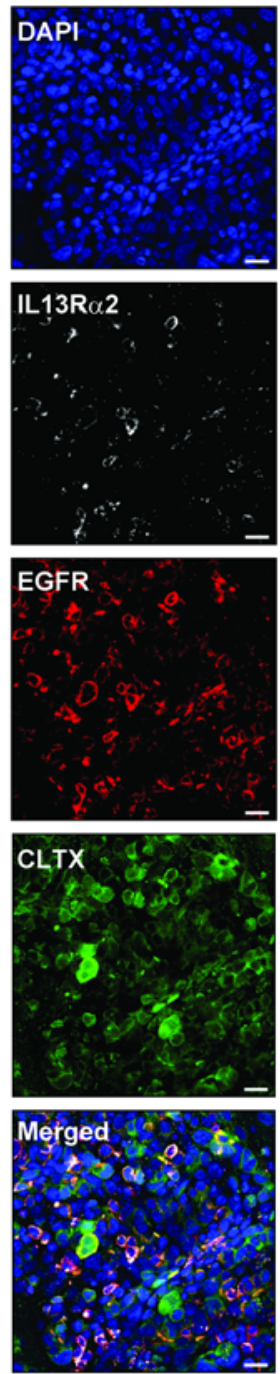

CD133

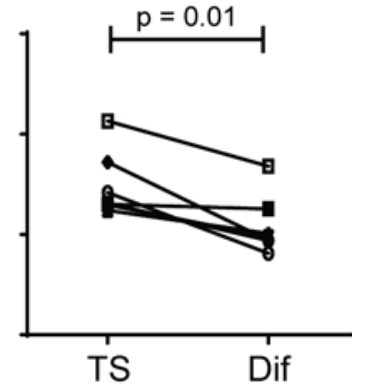

Fig. 1. CLTX binds broadly to primary GBM samples. (A) Freshly dispersed viable patient brain tumor (PBT) GBM cells (gated as DAPI-, CD45-, CD31-) were immunostained for expression of IL13Ra2, HER2, EGFR, or binding by Cy5.5-conjugated CLTX. Percentages of stained cells (blue) 
compared to control staining (grey) are indicated in each histogram. (B) Summary of staining results (\% positive as in (A)) for 22 primary PBTs (left) or 18 PBT tumor spheres (TS) (right). ND, not done. (C) Representative phenotype of a GBM xenograft established by stereotactic injection of 1 x $10^{5}$ PBT106 TS cells into the right forebrain of an NSG mouse. Tumor-bearing mouse brain was harvested 97 days after cell injection, and paraffin sections were stained with antibodies against IL13R $\alpha 2$, EGFR, and biotinconjugated CLTX. Staining was visualized by fluorochrome-conjugated secondary antibody staining and DAPI to identify nuclei. Scale bars: $20 \mu \mathrm{M}$. (D) Freshly-dispersed GBM samples separated into CD44+ and CD44- (left) or CD133+ and CD133- (right) subpopulations were examined for differences in CLTXCy5.5 staining, measured as mean fluorescence intensities (MFI). Only primary PBT samples with >20\% of CD44+ or CD133+ fractions were analyzed. (E) PBT-TS lines, maintained in neural stem cell medium (TS) or differentiation medium (Dif) for 14 days, were evaluated for CLTX-Cy5.5 and CD133 staining (MFI). (D, E) p values are indicated using paired two-way Student’s t-test. 
A

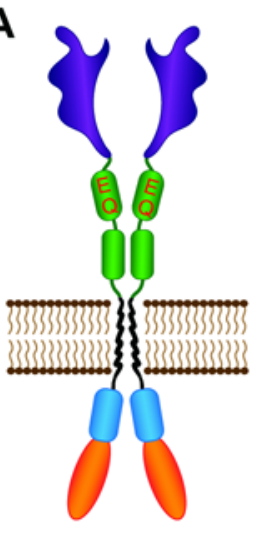

Tumor Targeting

CLTX

Spacer/Linker

$\lg \mathrm{G} 4-\mathrm{Fc}(\mathrm{EQ})$

CD4

Transmembrane

Costimulatory

CD28

Signaling

$\mathrm{CD} 3 \zeta$

\section{C}

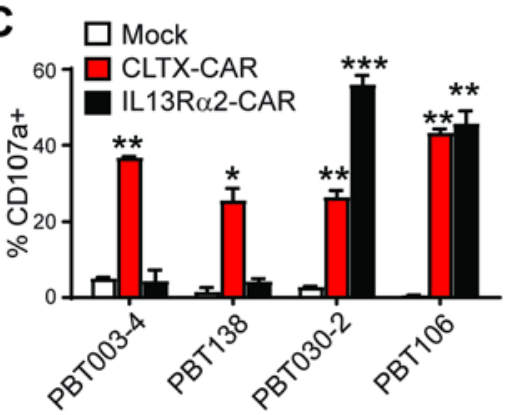

B
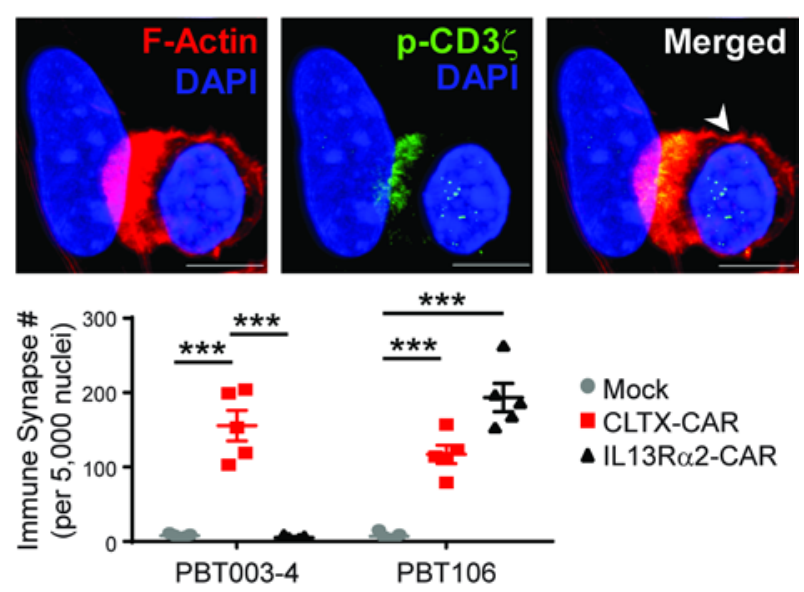

- Mock

- CLTX-CAR

$\triangle$ IL13R $\alpha 2-C A R$

D

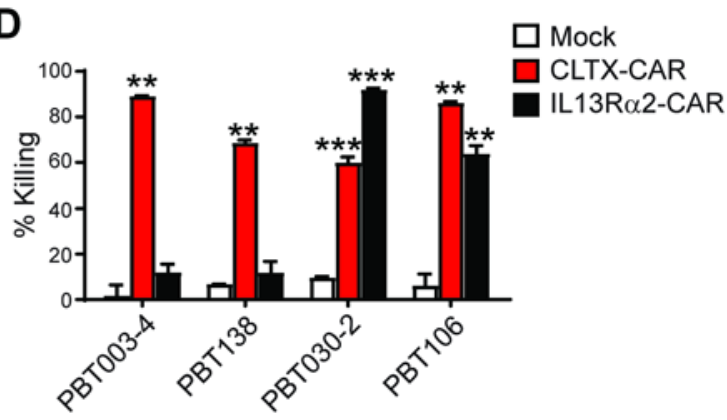

Fig. 2. Effector activity of CLTX-CAR T cells. (A) Diagram of a CAR containing a tumor targeting CLTX domain, an IgG4-Fc spacer domain with EQ mutations, a CD4 transmembrane domain, and intracellular signaling domains (CD28 and $\mathrm{CD} 3 \zeta)$. (B) Immunological synapse formation at $2 \mathrm{~h}$ after adding CLTX-CAR T cells to GBM cells dissociated from PBT003-4-TS (IL13Ra2-) or PBT106-TS (IL13R $\alpha 2+)$ (E:T=1:1). Top, a representative image of immunological synapse indicated by co-

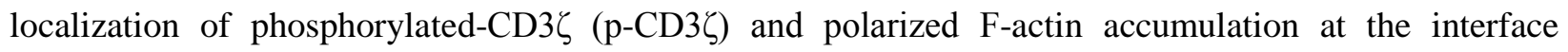

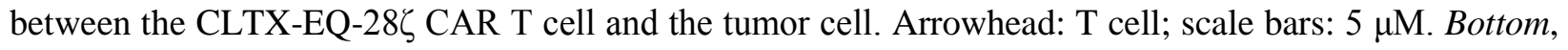
Number of immunological-synapses per 5,000 tumor nuclei, when mock-transduced T cells, CLTX-CAR T cells, or IL13R $\alpha 2-C A R$ T cells, were co-cultured with different GBM cells. Five different fields were counted for each group. Mean \pm SEM is plotted; ***, $\mathrm{p}<0.001$. (C) Degranulation of CLTX-CAR T cells (\% CD107a) after co-culture with GBM cells from different PBT-TS lines. Mock-transduced T cells, CLTXCAR T cells or IL13R $\alpha 2-C A R$ T cells were stimulated with either IL13R $\alpha 2$-negative (PBT003-4, PBT138) or IL13Ra2+ (PBT030-2, PBT106) GBM cells at a 1:1 E:T ratio for 5h, and CD3/CD19t+ gated 
cells were analyzed for surface CD107a expression as a marker of degranulation. Mean \pm S.E.M of \% CD107a+ cells in duplicate wells are depicted. (D) GBM cells were co-cultured with mock T cells, CLTX-CAR T cells or IL13R $\alpha 2-C A R$ T cells at an effector:target (E:T) ratio of 1:4 for $48 \mathrm{~h}$. CAR T cell activity is shown by the percentage of target cells eliminated. (C, D) *, $p<0.05 ;{ }^{* *}, p<0.01 ; * * *, p<0.001$ compared with mock T cells using one-way ANOVA with Bonferroni’s Multiple Comparison Tests. All PBT numbers indicate PBT-TS lines. 

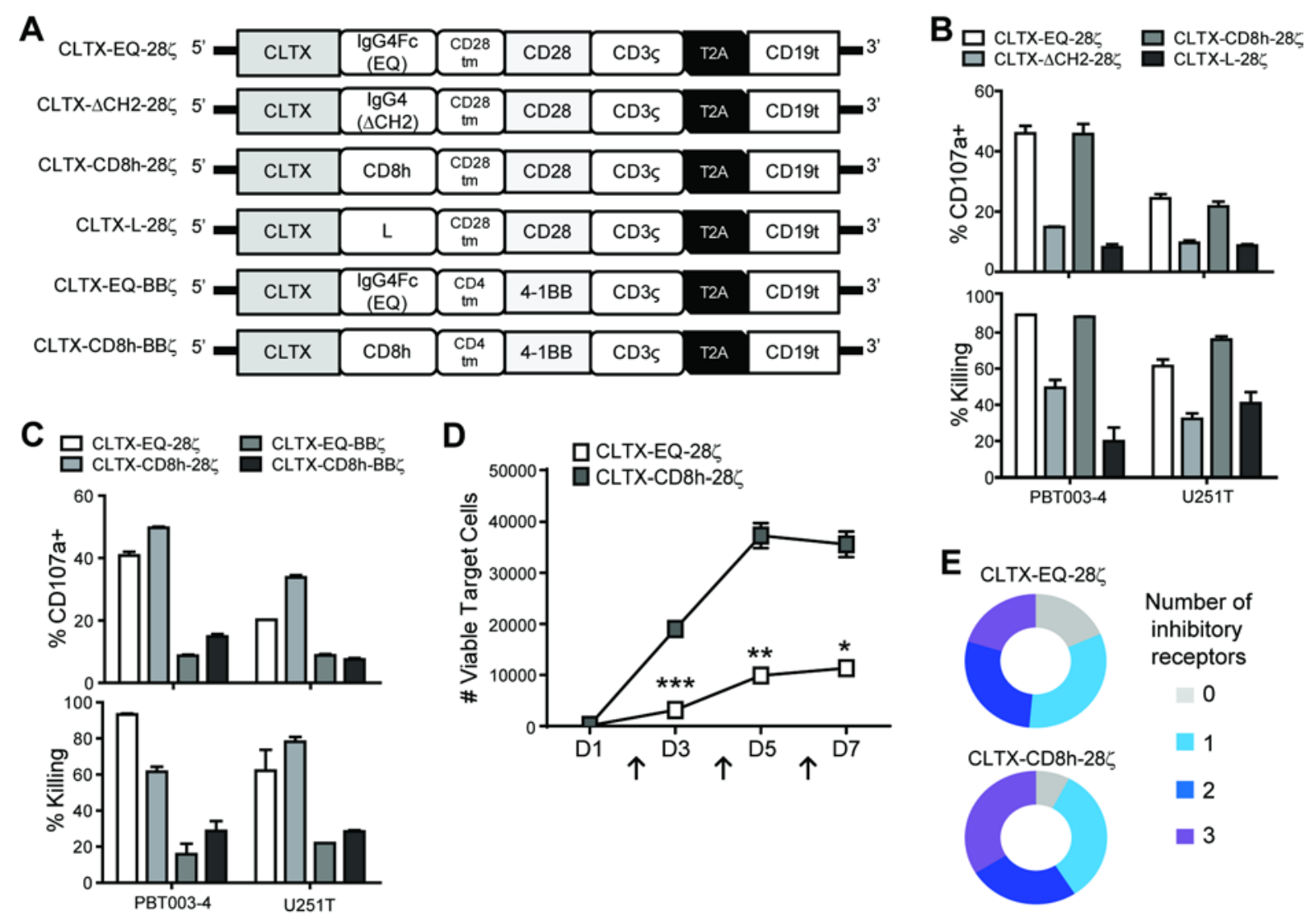

Fig. 3. Optimization of CLTX-CAR design for effector potency. (A) Schemas of CLTX-CARs bearing different spacers and/or co-stimulatory signals. (B) Degranulation (\% CD107a+; top) and cytotoxicity (\% target cell killing; bottom) for CLTX-CAR T cells with CD28 costimulatory domains but different spacers, tested against PBT003-4-TS or U251T GBM cells. (C) Degranulation (top) and cytotoxicity (bottom) of CLTX-CAR T cells with IgG4Fc(EQ) or CD8h spacers, and CD28 or 4-1BB co-stimulatory

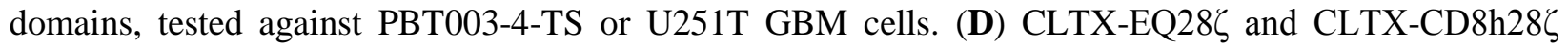
CAR T cells were co-cultured with PBT003-4-TS GBM cells (4,000 CAR+ cells, 16,000 tumor cells), and repetitively challenged with 32,000 tumor cells every 48 hours (arrows, D2, 4 and 6). Numbers of remaining viable tumor cells were quantified at the indicated time points during the rechallenge assay. Mean \pm SEM of duplicate wells are shown; *, $<<0.05,{ }^{* *}, \mathrm{p}<0.01$, and ***, $\mathrm{p}<0.001$ using an unpaired

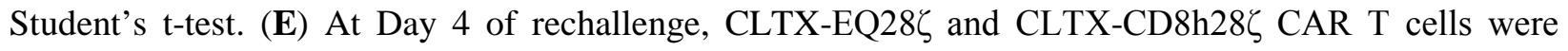


bioRxiv preprint doi: https://doi.org/10.1101/2020.01.24.918888; this version posted January 25, 2020. The copyright holder for this preprint (which was not certified by peer review) is the author/funder, who has granted bioRxiv a license to display the preprint in perpetuity. It is made available under aCC-BY-NC-ND 4.0 International license.

analyzed for T cell exhaustion markers and co-expression of PD-1, LAG-3 and TIM-3. All PBT numbers indicate PBT-TS lines. 
A
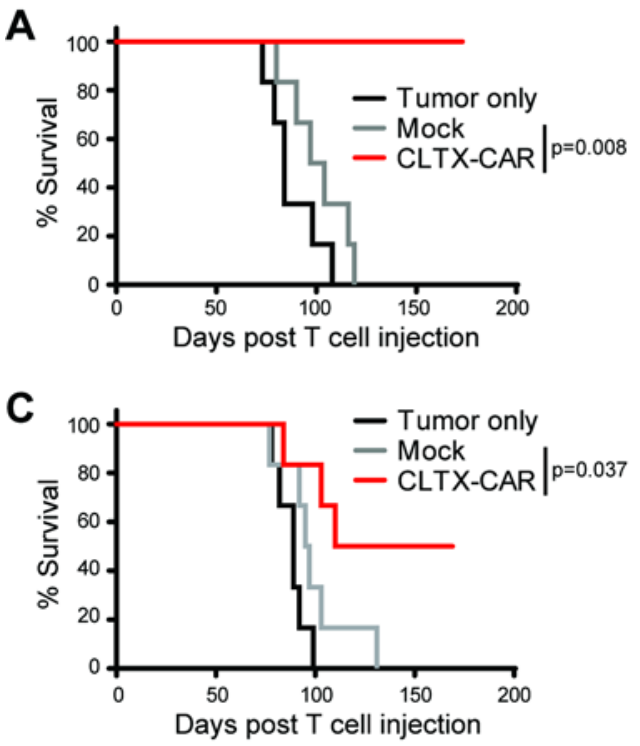

E

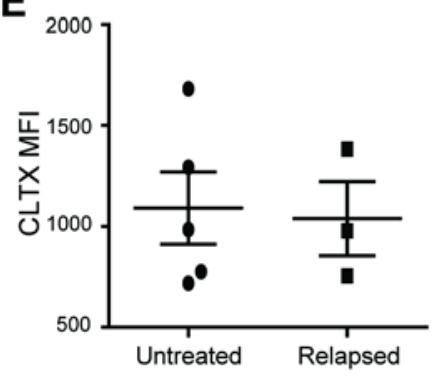

$\mathbf{F}$

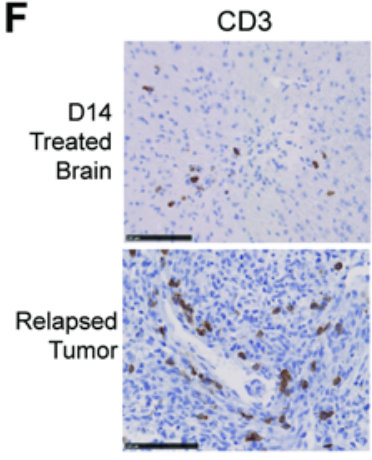

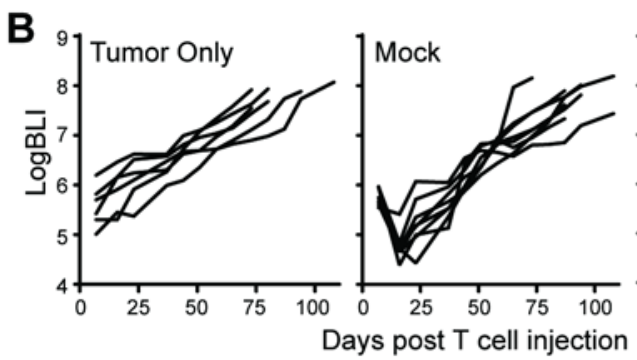
CLTX-CAR
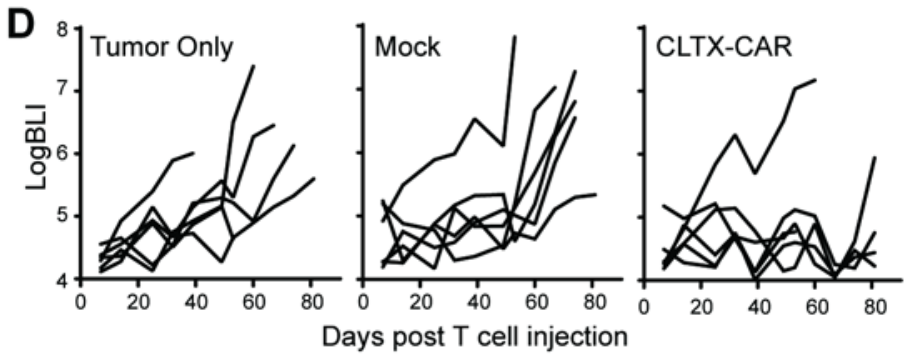

GranzymeB

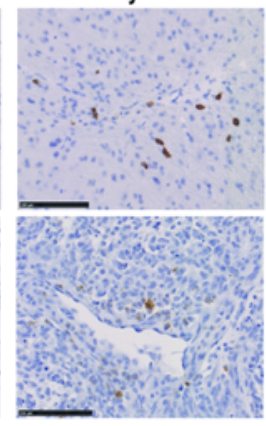

G

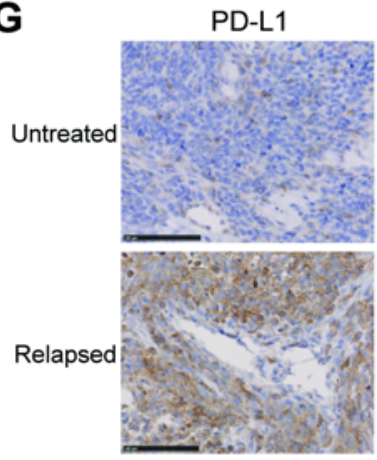

H

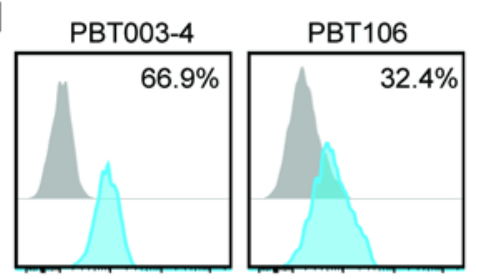

IFNYRA

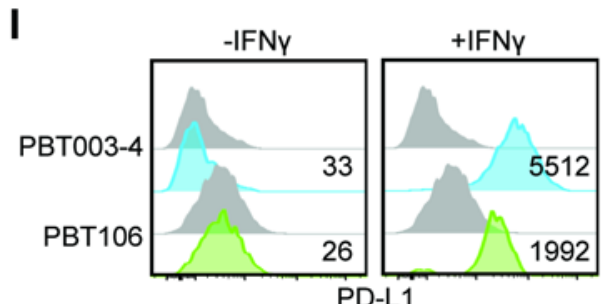

PD-L1

Fig. 4. In vivo antitumor activity of CLTX-CAR T cells. ffLuc ${ }^{+}$PBT106-TS (A,B) or PBT003-4-TS

(C,D) GBM cells were stereotactically implanted into the right forebrain of NSG mice $\left(1 \times 10^{5}\right.$ cells/mouse). On day 8 post tumor implantation, mice ( $n=6-7$ per group) received either no treatment

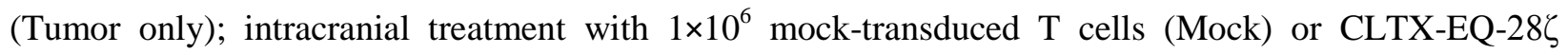
CAR+ T cells (CLTX-CAR). (A,C) Kaplan Meier survival analysis with Log-rank (Mantel Cox) test comparing CLTX-EQ28ל CAR T cell and Mock T cell treated groups. (B,D) Tumor volumes over time 
monitored using bioluminescent imaging. (E) Tumors harvested from NSG mice bearing PBT003-4-TS GBM xenografts that were either untreated $(n=5)$ or relapsed from those treated with CLTX-CAR T cells $(n=3)$, were dissociated into single cell suspensions and stained with CLTX-Cy5.5. Lines indicate mean MFI \pm SEM. (F) Immunochemical staining for CD3 and granzyme B on mouse brain sections at D14 post $\mathrm{T}$ cell injection and 7 days after tumor clearance (top), or on the relapsed tumor (bottom). (G) PD-L1 staining on untreated (top) or relapsed (bottom) PBT003-4-TS tumors. (H) Expression of IFN $\gamma$ Receptor A (IFN $\gamma$ RA) on GBM cells dissociated from PBT003-4-TS and PBT106-TS. Percentages of immunoreactive cells (blue) above that of isotype control staining (grey) are indicated in each histogram. (I) PD-L1 surface expression on PBT003-4-TS and PBT106-TS GBM cells with and without IFN $\gamma$ treatment. Numbers indicate PD-L1 MFI above isotype control. All PBT numbers indicate TS lines. 
A
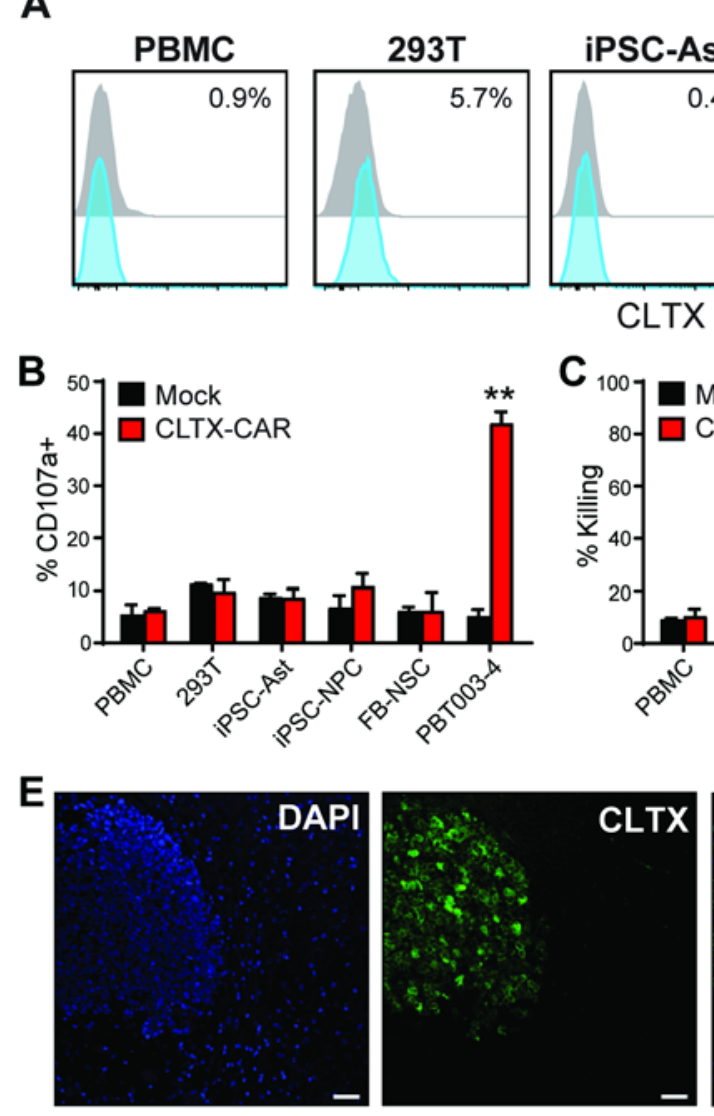

CLTX

C
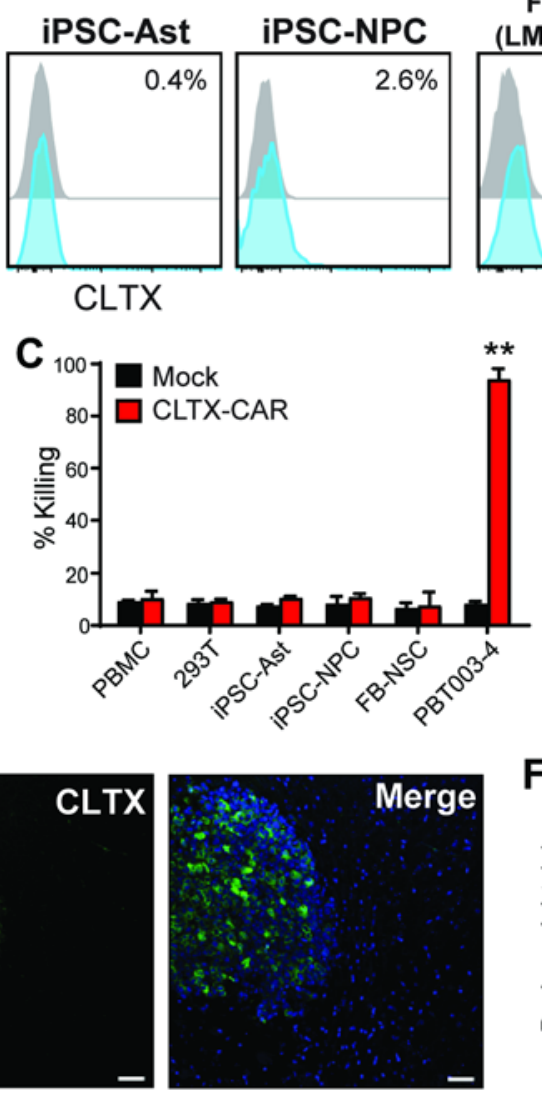

FB-NSC (LM-NSC008)
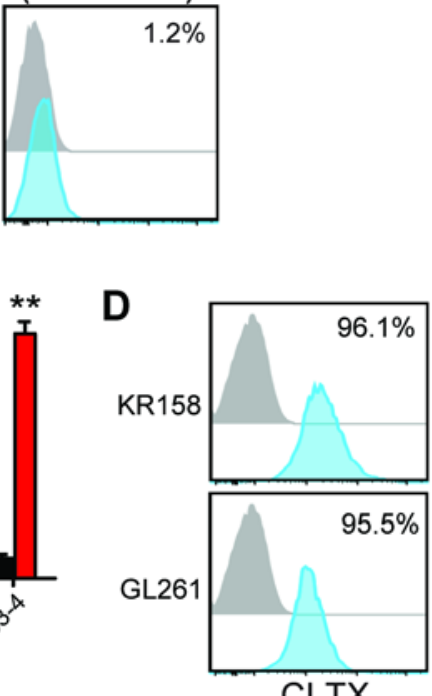

$\mathbf{F}$

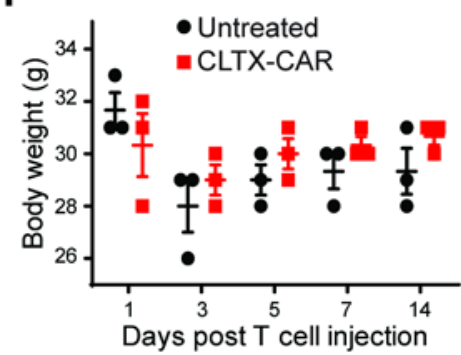

Fig. 5. Off-target evaluation of CLTX-CAR T cells. (A) CLTX-Cy5.5 staining on viable peripheral blood mononuclear cells (PBMC), human embryonic kidney 293T cells, iPSC-derived astrocytes (iPSCAst), neural progenitor cells (iPSC-NPC) or human fetal brain neural stem cells (FB-NSC line LMNSC008) evaluated by flow cytometry. Percentages of immunoreactive cells (blue) above control (grey) are indicated in each histogram. (B-C) Degranulation (B) and cytotoxicity (C) of mock-transduced (Mock)

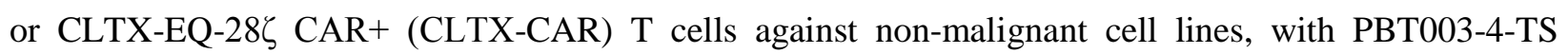
GBM cells as a positive control. Mean + SEM of duplicate wells are depicted; **, $\mathrm{p}<0.01$ when compared with mock-transduced T cells using an unpaired Student's t-test. (D) CLTX-Cy5.5 staining on mouse GBM cell lines. Percentages of positively-stained cells (blue) above that of isotype control (grey) are indicated in each histogram. (E) Representative immunofluorescent phenotype of a GBM xenograft established by stereotactically injecting $1 \times 10^{5}$ PBT106-TS cells into the right forebrain of an NSG mouse. 
The tumor-bearing mouse brain was harvested 93 days after cell injection, and paraffin sections were stained with DAPI to identify nuclei, and CLTX-Cy5.5 to depict the border between xenograft and normal mouse brain. Scale bars: $20 \mu \mathrm{m}$. (F) Body weights of NSG mice receiving intravenous administration of $5 \times 10^{7}$ CLTX-EQ-28 $\zeta$ CAR + (CLTX-CAR) T cells. Body weights were monitored over 2 weeks and compared with untreated NSG mice. Lines indicate mean \pm SEM. All PBT numbers indicate TS lines. 
A
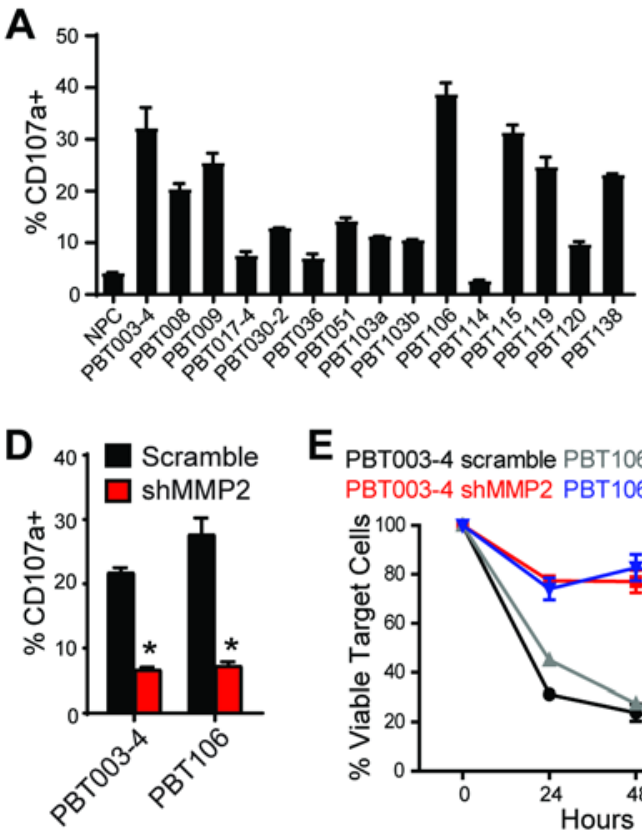

$E_{P}$

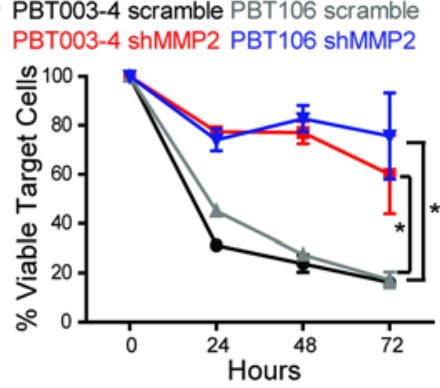
PBT003-4 shMMP2 PBT106 shMMP2

G

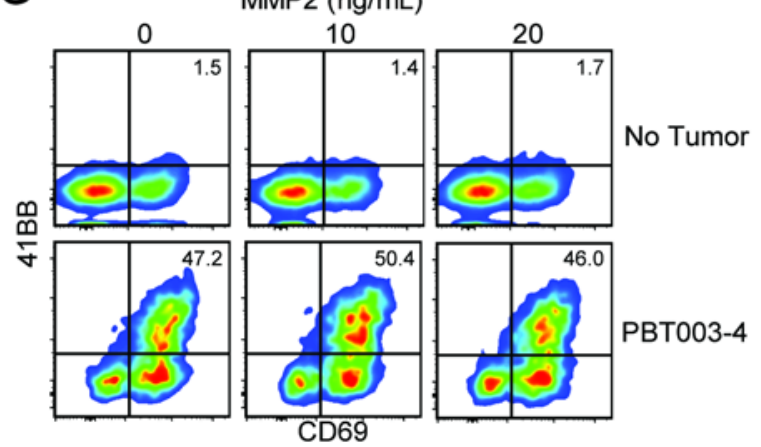

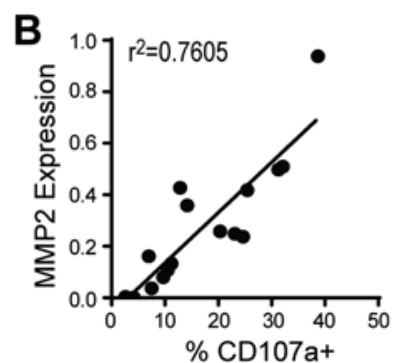

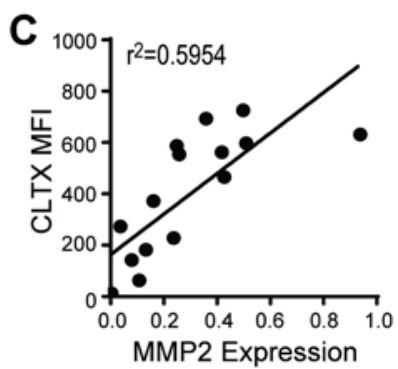

F

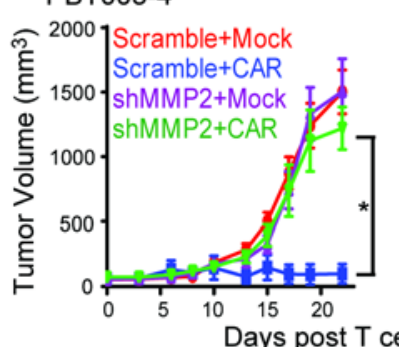

PBT106
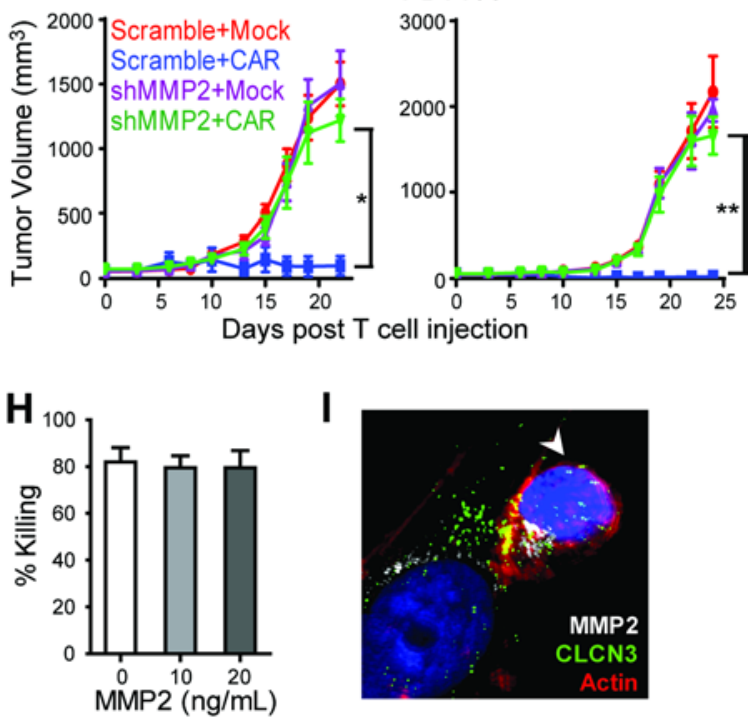

Fig. 6. CLTX-CAR T cell effector activity requires MMP2 expression on target cells. (A) Degranulation of CLTX-EQ-28ל CAR+ T cells against iPSC-NPCs and GBM cells from indicated TS lines. Shown are mean \pm SEM of duplicate wells. (B-C) MMP2 mRNA expression $\left(2^{-4 \mathrm{Ct}}\right.$ compared with ACTIN) in the GBM-TS lines were compared to their corresponding stimulation of CLTX-CAR T cell degranulation (B) and CLTX-Cy5.5 staining (C); with correlation coefficients $\left(\mathrm{r}^{2}\right)$ indicated in each graph. (D) Degranulation of CLTX-CAR T cells tested against PBT003-4-TS or PBT106-TS lines that had been transduced with scramble shRNA or shRNA targeting MMP2 (shMMP2). (E) Cytotolytic activity of CLTX-CAR T cells against scramble shRNA or shMMP2 transduced GBM cells over a 72h co-culture $(\mathrm{E}: \mathrm{T}=1: 4) .(\mathbf{D}, \mathbf{E}) * \mathrm{p}<0.05$ when compared with scramble shRNA transduced targets using an unpaired 
Student's t-test. (F) Scramble shRNA or shMMP2 transduced GBM cells $\left(5 \times 10^{6}\right)$ were injected subcutaneously into the right flank of NSG mice. Mock-transduced or CLTX-EQ-28ל CAR+ T cells $\left(3 \times 10^{6}\right)$ were then injected into the tumors at day 14 (tumor diamater $\sim 5 \mathrm{~mm}$ ), and tumor size was monitored over time with caliper measurements. ${ }^{*} \mathrm{p}<0.05,{ }^{* *} \mathrm{p}<0.01$ using one-way ANOVA with Bonferroni's Multiple Comparison Tests. (G) CLTX-CAR T cells were cultured with indicated concentrations of soluble MMP2 in the absence (top row) or presence (bottom row) of PBT003-4 GBM cells (E:T=1:4) for 24h. T cell activation was determined by flow cytometric analysis of 4-1BB and CD69 surface expression. Quadrants were drawn based on control staining, and percentages of double-staining cells are indicated in each histogram. (H) Elimination (\% killing) of PBT003-4-TS GBM cells by CLTXCAR T cells after $48 \mathrm{~h}$ of co-culture $(\mathrm{E}: \mathrm{T}=1: 4)$ at different concentrations of soluble MMP2. Percentages of tumor killing were calculated using the numbers of viable tumor cells when cultured in the absence of CLTX-CAR T cells; mean \pm SEM of duplicate wells are shown. (I) Immunofluorescence staining of MMP2, CLCN3 and actin at $2 \mathrm{~h}$ after initiating co-culture of CLTX-CAR T cells and PBT003-4 GBM cells (E:T=1:1), arrowhead: T cell. All PBT numbers indicate TS lines. 